\title{
Evolution of states in a continuum migration model
}

\author{
Yuri Kondratiev ${ }^{1,2}$. Yuri Kozitsky ${ }^{3}$
}

Received: 5 November 2016 / Revised: 6 February 2017 / Accepted: 20 February 2017 /

Published online: 1 March 2017

(C) The Author(s) 2017. This article is published with open access at Springerlink.com

\begin{abstract}
The Markov evolution of states of a continuum migration model is studied. The model describes an infinite system of entities placed in $\mathbb{R}^{d}$ in which the constituents appear (immigrate) with rate $b(x)$ and disappear, also due to competition. For this model, we prove the existence of the evolution of states $\mu_{0} \mapsto \mu_{t}$ such that the moments $\mu_{t}\left(N_{\Lambda}^{n}\right), n \in \mathbb{N}$, of the number of entities in compact $\Lambda \subset \mathbb{R}^{d}$ remain bounded for all $t>0$. Under an additional condition, we prove that the density of entities and the second correlation function remain point-wise bounded globally in time.
\end{abstract}

Keywords Markov evolution · Competition kernel · Poisson random field

Mathematics Subject Classification $60 \mathrm{~J} 80 \cdot 92 \mathrm{D} 25 \cdot 82 \mathrm{C} 22$

\section{Introduction}

We study the Markov dynamics of an infinite system of point entities placed in $\mathbb{R}^{d}$, $d \geq 1$, which appear (immigrate) with space-dependent rate $b(x) \geq 0$, and disappear. The rate of disappearance of the entity located at a given $x \in \mathbb{R}^{\bar{d}}$ is the sum of the

Yuri Kozitsky

jkozi@hektor.umcs.lublin.pl

Yuri Kondratiev

kondrat@math.uni-bielefeld.de

1 Fakutät für Mathematik, Universität Bielefeld, 33615 Bielefeld, Germany

2 Interdisciplinary Center for Complex Systems, Dragomanov University, Kyiv, Ukraine

3 Instytut Matematyki, Uniwersytet Marii Curie-Skłodowskiej, 20-031 Lublin, Poland

Birkhäuser 
intrinsic disappearance rate $m(x) \geq 0$ and the part related to the interaction with the existing community, which is interpreted as competition between the entities. The phase space is the set $\Gamma$ of all subsets $\gamma \subset \mathbb{R}^{d}$ such that the set $\gamma_{\Lambda}:=\gamma \cap \Lambda$ is finite whenever $\Lambda \subset \mathbb{R}^{d}$ is compact. For each such $\Lambda$, one defines the counting map $\Gamma \ni \gamma \mapsto\left|\gamma_{\Lambda}\right|:=\#\{\gamma \cap \Lambda\}$, where the latter denotes cardinality. Thereby, one introduces the subsets $\Gamma^{\Lambda, n}:=\left\{\gamma \in \Gamma:\left|\gamma_{\Lambda}\right|=n\right\}, n \in \mathbb{N}_{0}$, and equips $\Gamma$ with the $\sigma$-field generated by all such $\Gamma^{\Lambda, n}$. This allows for considering probability measures on $\Gamma$ as states of the system. Among them there are Poissonian states in which the entities are independently distributed over $\mathbb{R}^{d}$, see [6, Chapter 2]. They may serve as reference states for studying correlations between the positions of the entities. For the nonhomogeneous Poisson measure $\pi_{\varrho}$ with density $\varrho: \mathbb{R}^{d} \rightarrow \mathbb{R}_{+}:=[0,+\infty)$, $n \in \mathbb{N}_{0}$, and every compact $\Lambda$, one has

$$
\pi_{\varrho}\left(\Gamma^{\Lambda, n}\right)=\langle\varrho\rangle_{\Lambda}^{n} \exp \left(-\langle\varrho\rangle_{\Lambda}\right) / n !, \quad\langle\varrho\rangle_{\Lambda}:=\int_{\Lambda} \varrho(x) d x
$$

which implies

$$
\pi_{\varrho}\left(N_{\Lambda}\right)=\int_{\Gamma}\left|\gamma_{\Lambda}\right| \pi_{\varrho}(d \gamma)=\langle\varrho\rangle_{\Lambda}
$$

where $N_{\Lambda}$ is the number of entities contained in $\Lambda$. In the case of $\varrho \equiv \varkappa>0$, one deals with the homogeneous Poisson measure $\pi_{\varkappa}$.

The counting map $\Gamma \ni \gamma \mapsto|\gamma|$ can also be defined for $\Lambda=\mathbb{R}^{d}$. Then the set of finite configurations

$$
\Gamma_{0}:=\bigcup_{n \in \mathbb{N}_{0}}\{\gamma \in \Gamma:|\gamma|=n\}
$$

is clearly measurable. In a state with the property $\mu\left(\Gamma_{0}\right)=1$, the system is ( $\mu$-almost surely) finite. By (1.1) one gets that either $\pi_{\varrho}\left(\Gamma_{0}\right)=1$ or $\pi_{\varrho}\left(\Gamma_{0}\right)=0$, depending on whether or not $\varrho$ is globally integrable. If $\pi_{\varkappa}\left(\Gamma_{0}\right)=0$, the system in state $\pi_{\varkappa}$ is infinite. The use of infinite configurations for modeling large finite populations is as a rule justified, see, e.g., [2], by the argument that in such a way one gets rid of the boundary and size effects. Note that a finite system with dispersal-like the one studied in [5,7]—being placed in a noncompact habitat always disperse to fill its empty parts, and thus is developing. Infinite configurations are supposed to model developed populations. In this work, we shall consider infinite systems and hence deal with states $\mu$ such that $\mu\left(\Gamma_{0}\right)=0$.

To characterize states on $\Gamma$ one employs observables-appropriate functions $F$ : $\Gamma \rightarrow \mathbb{R}$. Their evolution is obtained from the Kolmogorov equation

$$
\frac{d}{d t} F_{t}=L F_{t},\left.\quad F_{t}\right|_{t=0}=F_{0}, \quad t>0,
$$

where the generator $L$ specifies the model. The states' evolution is then obtained from the Fokker-Planck equation 


$$
\frac{d}{d t} \mu_{t}=L^{*} \mu_{t},\left.\quad \mu_{t}\right|_{t=0}=\mu_{0},
$$

related to that in (1.4) by the duality $\mu_{t}\left(F_{0}\right)=\mu_{0}\left(F_{t}\right)$, where

$$
\mu(F):=\int_{\Gamma} F(\gamma) \mu(d \gamma) .
$$

The model that we study in this work is specified by the following

$$
\begin{aligned}
(L F)(\gamma)= & \sum_{x \in \gamma}\left(m(x)+\sum_{y \in \gamma \backslash x} a(x-y)\right)[F(\gamma \backslash x)-F(\gamma)] \\
& +\int_{\mathbb{R}^{d}} b(x)[F(\gamma \cup x)-F(\gamma)] d x .
\end{aligned}
$$

Here, for appropriate $\gamma \in \Gamma$ and $x \in \mathbb{R}^{d}$, by writing $\gamma \backslash x$ we mean $\gamma \backslash\{x\}$. Likewise, $\gamma \cup x$ stands for $\gamma \cup\{x\}$. In (1.6), $b(x)$ is the immigration rate, $m(x) \geq 0$ is the intrinsic emigration (mortality) rate, and $a \geq 0$ is the competition kernel. The model parameters are supposed to satisfy the following.

Assumption 1.1 The competition kernel $a$ is continuous and belongs to $L^{1}\left(\mathbb{R}^{d}\right) \cap$ $L^{\infty}\left(\mathbb{R}^{d}\right)$. If not explicitly stated otherwise, $a(0)>0$. The immigration and mortality rates $b$ and $m$ are continuous and bounded.

According to this we set

$$
\begin{aligned}
\langle a\rangle & =\int_{\mathbb{R}^{d}} a(x) d x, \quad\|a\|=\sup _{x \in \mathbb{R}^{d}} a(x), \\
\|b\| & =\sup _{x \in \mathbb{R}^{d}} b(x), \quad\|m\|=\sup _{x \in \mathbb{R}^{d}} m(x) .
\end{aligned}
$$

If one sets in (1.6) $a \equiv 0$, the model becomes exactly soluble, see Sect. 2.3 below. This means that the evolution can be constructed explicitly for each initial state $\mu_{0}$. In this case, assuming that $\mu_{0}\left(N_{\Lambda}^{n}\right)<\infty$ for all $n \in \mathbb{N}$, one can get the information about the time dependence of such moments. Namely, if $m(x) \geq m_{*}>0$ for all $x \in \mathbb{R}^{d}$, then for each compact $\Lambda$, the following holds

$$
\forall t>0 \quad \mu_{t}\left(N_{\Lambda}^{n}\right) \leq C_{\Lambda}^{(n)}
$$

Otherwise, all the moments $\mu_{t}\left(N_{\Lambda}^{n}\right)$ are increasing ad infinitum as $t \rightarrow+\infty$. If the initial state is $\pi_{\varrho_{0}}$, then $\mu_{t}=\pi_{\varrho_{t}}$ with $\varrho_{t}(x)=\varrho_{0}(x)+b(x) t$ for all $x$ such that $m(x)=0, \operatorname{cf}(2.22)$ below. In [3], for the model (1.6) with $m \equiv 0$ and a nonzero $a$ satisfying a certain (quite burdensome) condition, it was shown that $\mu_{t}\left(N_{\Lambda}\right) / \mathrm{V}(\Lambda) \leq$ $C$ for large enough values of the Euclidean volume $\mathrm{V}(\Lambda)$, provided the evolution of states $\mu_{0} \mapsto \mu_{t}$ exists. 
In this article, assuming that the initial state $\mu_{0}$ is sub-Poissonian, see Definition 2.1 below, we prove that the evolution of states $\mu_{0} \mapsto \mu_{t}, t>0$, exists (Theorem 2.4) and is such that $\mu_{t}\left(N_{\Lambda}^{n}\right) \leq C_{\Lambda}^{(n)}$ for each $t>0$ (Theorem 2.5) and all $m$, including the case $m \equiv 0$. Moreover, if the correlation functions $k_{\mu_{0}}^{(n)}, n \in \mathbb{N}$, of the initial state are continuous, see Sect. 2.1 below, then $k_{\mu_{t}}^{(n)}, n \in \mathbb{N}$, are also continuous and the following holds

$$
k_{\mu_{t}}^{(1)}(x) \leq C_{1}, \quad k_{\mu_{t}}^{(2)}(x, y) \leq C_{2},
$$

with some positive $C_{1}$ and $C_{2}$.

The structure of the article is as follows. In Sect. 2, we introduce the necessary technicalities and then formulate the results: Theorems 2.4 and 2.5. Thereafter, we make a number of comments to them. In Sects. 3 and 4, we present the proofs of Theorems 2.4 and 2.5 , respectively.

\section{Preliminaries and the results}

We begin by presenting some facts on the subject - a more detailed description of them can be found in $[5,7,8]$ and in the literature quoted therein.

By $\mathcal{B}\left(\mathbb{R}^{d}\right)$ and $\mathcal{B}_{\mathrm{b}}\left(\mathbb{R}^{d}\right)$ we denote the sets of all Borel and bounded Borel subsets of $\mathbb{R}^{d}$, respectively. The configuration space $\Gamma$ is equipped with the vague topology, see [8], and thus with the corresponding Borel $\sigma$-field $\mathcal{B}(\Gamma)$, which makes it a standard Borel space. Note that $\mathcal{B}(\Gamma)$ is exactly the $\sigma$-field generated by the sets $\Gamma^{\Lambda, n}$, mentioned in Introduction. By $\mathcal{P}(\Gamma)$ we denote the set of all probability measures on $(\Gamma, \mathcal{B}(\Gamma))$.

\subsection{Correlation functions}

Like in [5,7], the evolution of states will be described by means of correlation functions without the direct use of (1.5). To explain the essence of this approach let us consider the set $\Theta$ of all compactly supported continuous functions $\theta: \mathbb{R}^{d} \rightarrow(-1,0]$. For a state, $\mu$, its Bogoliubov functional, cf. [9,12], is

$$
B_{\mu}(\theta)=\int_{\Gamma} \prod_{x \in \gamma}(1+\theta(x)) \mu(d \gamma), \quad \theta \in \Theta
$$

For the homogeneous Poisson measure $\pi_{\varkappa}$, it takes the form

$$
B_{\pi_{\varkappa}}(\theta)=\exp \left(\varkappa \int_{\mathbb{R}^{d}} \theta(x) d x\right) .
$$

Definition 2.1 The set of sub-Poissonian states $\mathcal{P}_{\exp }(\Gamma)$ consists of all those states $\mu \in \mathcal{P}(\Gamma)$ for which $B_{\mu}$ can be continued, as a function of $\theta$, to an exponential type entire function on $L^{1}\left(\mathbb{R}^{d}\right)$. 
It can be shown that a given $\mu$ belongs to $\mathcal{P}_{\exp }(\Gamma)$ if and only if its functional $B_{\mu}$ can be written down in the form

$$
B_{\mu}(\theta)=1+\sum_{n=1}^{\infty} \frac{1}{n !} \int_{\left(\mathbb{R}^{d}\right)^{n}} k_{\mu}^{(n)}\left(x_{1}, \ldots, x_{n}\right) \theta\left(x_{1}\right) \cdots \theta\left(x_{n}\right) d x_{1} \cdots d x_{n}
$$

where $k_{\mu}^{(n)}$ is the $n$-th order correlation function of $\mu$, which is a symmetric element of $L^{\infty}\left(\left(\mathbb{R}^{d}\right)^{n}\right)$ satisfying

$$
\left\|k_{\mu}^{(n)}\right\|_{L^{\infty}\left(\left(\mathbb{R}^{d}\right)^{n}\right)} \leq C \exp (\vartheta n), \quad n \in \mathbb{N}_{0}
$$

with some $C>0$ and $\vartheta \in \mathbb{R}$. Note that $k_{\pi_{\varkappa}}^{(n)}\left(x_{1}, \ldots, x_{n}\right)=\varkappa^{n}$. Note also that (2.2) resembles the Taylor expansion of the characteristic function of a probability measure. In view of this, $k_{\mu}^{(n)}$ are also called (factorial) moment functions, cf. e.g., [11].

Recall that $\Gamma_{0}$ - the set of all finite $\gamma \in \Gamma$ defined in (1.3) - is an element of $\mathcal{B}(\Gamma)$. A function $G: \Gamma_{0} \rightarrow \mathbb{R}$ is $\mathcal{B}(\Gamma) / \mathcal{B}(\mathbb{R})$-measurable, see [5], if and only if, for each $n \in \mathbb{N}$, there exists a symmetric Borel function $G^{(n)}:\left(\mathbb{R}^{d}\right)^{n} \rightarrow \mathbb{R}$ such that

$$
G(\eta)=G^{(n)}\left(x_{1}, \ldots, x_{n}\right), \text { for } \eta=\left\{x_{1}, \ldots, x_{n}\right\}
$$

Definition 2.2 A measurable function $G: \Gamma_{0} \rightarrow \mathbb{R}$ is said to have bounded support if: (a) there exists $\Lambda \in \mathcal{B}_{\mathrm{b}}\left(\mathbb{R}^{d}\right)$ such that $G(\eta)=0$ whenever $\eta \cap\left(\mathbb{R}^{d} \backslash \Lambda\right) \neq \emptyset$; (b) there exists $N \in \mathbb{N}_{0}$ such that $G(\eta)=0$ whenever $|\eta|>N$. By $\Lambda(G)$ and $N(G)$ we denote the smallest $\Lambda$ and $N$ with the properties just mentioned. By $B_{\mathrm{bs}}\left(\Gamma_{0}\right)$ we denote the set of all bounded functions with bounded support.

The Lebesgue-Poisson measure $\lambda$ on $\left(\Gamma_{0}, \mathcal{B}\left(\Gamma_{0}\right)\right)$ is defined by the following formula

$$
\int_{\Gamma_{0}} G(\eta) \lambda(d \eta)=G(\emptyset)+\sum_{n=1}^{\infty} \frac{1}{n !} \int_{\left(\mathbb{R}^{d}\right)^{n}} G^{(n)}\left(x_{1}, \ldots, x_{n}\right) d x_{1} \cdots d x_{n}
$$

holding for all $G \in B_{\mathrm{bs}}\left(\Gamma_{0}\right)$. Like in (2.4), we introduce $k_{\mu}: \Gamma_{0} \rightarrow \mathbb{R}$ such that $k_{\mu}(\eta)=k_{\mu}^{(n)}\left(x_{1}, \ldots, x_{n}\right)$ for $\eta=\left\{x_{1}, \ldots, x_{n}\right\}, n \in \mathbb{N}$. We also set $k_{\mu}(\emptyset)=1$. With the help of the measure introduced in (2.5), the expressions for $B_{\mu}$ in (2.1) and (2.2) can be combined into the following formulas

$$
\begin{aligned}
B_{\mu}(\theta) & =\int_{\Gamma_{0}} k_{\mu}(\eta) \prod_{x \in \eta} \theta(x) \lambda(d \eta)=: \int_{\Gamma_{0}} k_{\mu}(\eta) e(\eta ; \theta) \lambda(d \eta) \\
& =\int_{\Gamma} \prod_{x \in \gamma}(1+\theta(x)) \mu(d \gamma)=: \int_{\Gamma} F_{\theta}(\gamma) \mu(d \gamma) .
\end{aligned}
$$


Thereby, one can transform the action of $L$ on $F$, see (1.6), to the action of $L^{\Delta}$ on $k_{\mu}$ according to the rule

$$
\int_{\Gamma}\left(L F_{\theta}\right)(\gamma) \mu(d \gamma)=\int_{\Gamma_{0}}\left(L^{\Delta} k_{\mu}\right)(\eta) e(\eta ; \theta) \lambda(d \eta) .
$$

This will allow us to pass from (1.5) to the corresponding Cauchy problem for the correlation functions

$$
\frac{d}{d t} k_{t}=L^{\Delta} k_{t},\left.\quad k_{t}\right|_{t=0}=k_{\mu_{0}} .
$$

By (2.7) the action of $L^{\Delta}$ is as follows

$$
\left(L^{\Delta} k\right)(\eta)=\left(L^{\Delta,-} k\right)(\eta)+\sum_{x \in \eta} b(x) k(\eta \backslash x),
$$

where

$$
\left(L^{\Delta,-} k\right)(\eta)=-E(\eta) k(\eta)-\int_{\mathbb{R}^{d}}\left(\sum_{y \in \eta} a(x-y)\right) k(\eta \cup x) d x,
$$

and

$$
E(\eta)=\sum_{x \in \eta} m(x)+\sum_{x \in \eta} \sum_{y \in \eta \backslash x} a(x-y) .
$$

In the next subsection, we introduce the spaces where we are going to define (2.8).

\subsection{The Banach spaces}

By (2.2) and (2.6), it follows that $\mu \in \mathcal{P}_{\exp }(\Gamma)$ implies

$$
\left|k_{\mu}(\eta)\right| \leq C \exp (\vartheta|\eta|)
$$

holding for $\lambda$-almost all $\eta \in \Gamma_{0}$, some $C>0$, and $\vartheta \in \mathbb{R}$. In view of this, we set

$$
\mathcal{K}_{\vartheta}:=\left\{k: \Gamma_{0} \rightarrow \mathbb{R}:\|k\|_{\vartheta}<\infty\right\}
$$

where

$$
\|k\|_{\vartheta}=\operatorname{ess}_{\sup } \operatorname{si}_{0}\left\{\left|k_{\mu}(\eta)\right| \exp (-\vartheta|\eta|)\right\} .
$$

Clearly, (2.12) and (2.13) define a Banach space with the usual point-wise linear operations. In the following, we use the ascending scale of such spaces $\mathcal{K}_{\vartheta}, \vartheta \in \mathbb{R}$, with the property

$$
\mathcal{K}_{\vartheta} \hookrightarrow \mathcal{K}_{\vartheta^{\prime}}, \quad \vartheta<\vartheta^{\prime}
$$

where $\hookrightarrow$ denotes continuous embedding. 
For $G \in B_{\mathrm{bs}}(\Gamma)$, we set

$$
(K G)(\gamma)=\sum_{\eta \Subset \gamma} G(\eta),
$$

where $\Subset$ indicates that the summation is taken over all finite subsets. It satisfies, see Definition 2.2,

$$
|(K G)(\gamma)| \leq C_{G}(1+|\gamma \cap \Lambda(G)|)^{N(G)}, \quad C_{G}:=\sup _{\eta \in \Gamma_{0}}|G(\eta)| .
$$

The latter means that $\mu(K G)<\infty$ for each $\mu \in \mathcal{P}_{\exp }(\Gamma)$. By (2.6) this yields

$$
\left\langle\left\langle G, k_{\mu}\right\rangle\right\rangle:=\int_{\Gamma_{0}} G(\eta) k_{\mu}(\eta) \lambda(d \eta)=\mu(K G)<\infty .
$$

Set

$$
B_{\mathrm{bs}}^{\star}\left(\Gamma_{0}\right)=\left\{G \in B_{\mathrm{bs}}\left(\Gamma_{0}\right):(K G)(\gamma) \geq 0 \text { for all } \gamma \in \Gamma\right\} .
$$

By [8, Theorems 6.1 and 6.2 and Remark 6.3] one can prove the next statement.

Proposition 2.3 Let a measurable function $k: \Gamma_{0} \rightarrow \mathbb{R}$ have the following properties:

(a) $\left\langle\langle G, k\rangle \geq \geq 0, \quad\right.$ for all $G \in B_{\mathrm{bs}}^{\star}\left(\Gamma_{0}\right)$;

(b) $k(\emptyset)=1 ; \quad(c) k(\eta) \leq C^{|\eta|}$,

with (c) holding for some $C>0$ and $\lambda$-almost all $\eta \in \Gamma_{0}$. Then there exists a unique state $\mu \in \mathcal{P}_{\exp }(\Gamma)$ for which $k$ is the correlation function.

Define, cf. (2.17),

$$
\mathcal{K}_{\vartheta}^{\star}=\left\{k \in \mathcal{K}_{\vartheta}:\left\langle\langle G, k\rangle \geq 0 \text { for all } G \in B_{\mathrm{bs}}^{\star}\left(\Gamma_{0}\right)\right\}\right.
$$

This is clearly a subset of the cone

$$
\mathcal{K}_{\vartheta}^{+}=\left\{k \in \mathcal{K}_{\vartheta}: k(\eta) \geq 0 \text { for } \lambda-\text { almost all } \eta \in \Gamma_{0}\right\}
$$

By Proposition 2.3 it follows that each $k \in \mathcal{K}_{\vartheta}^{\star}$ with the property $k(\emptyset)=1$ is the correlation function of a unique state $\mu \in \mathcal{P}_{\exp }(\Gamma)$. Then we define

$$
\mathcal{K}=\bigcup_{\vartheta \in \mathbb{R}} \mathcal{K}_{\vartheta}, \quad \mathcal{K}^{\star}=\bigcup_{\vartheta \in \mathbb{R}} \mathcal{K}_{\vartheta}^{\star}
$$

As a sum of Banach spaces, the linear space $\mathcal{K}$ is equipped with the corresponding inductive topology that turns it into a locally convex space. 


\subsection{Without competition}

The version of (1.6) with $a \equiv 0$ is known as the Surgailis model, see [14] and the discussion in [3]. This model is exactly soluble. This means that the solution of (2.8) can be written down explicitly in the following form

$$
k_{t}(\eta)=\sum_{\xi \subset \eta} e\left(\xi ; \phi_{t}\right) e\left(\eta \backslash \xi ; \psi_{t}\right) k_{\mu_{0}}(\eta \backslash \xi),
$$

where

$$
\begin{aligned}
& \psi_{t}(x)=e^{-m(x) t}, \quad e(\xi ; \phi)=\prod_{x \in \xi} \phi(x), \\
& \phi_{t}(x)= \begin{cases}\left(1-e^{-m(x) t}\right) \frac{b(x)}{m(x)} & \text { for } m(x)>0, \\
b(x) t & \text { for } m(x)=0 .\end{cases}
\end{aligned}
$$

The corresponding state $\mu_{t}$ has the Bogoliubov functional

$$
B_{\mu_{t}}(\theta)=\exp \left(\int_{\mathbb{R}^{d}} \theta(x) \phi_{t}(x) d x\right) B_{\mu_{0}}\left(\theta \psi_{t}\right),
$$

which one obtains from (2.6) and (2.21). This formula can be used to extend the evolution $\mu_{0} \mapsto \mu_{t}$ to all $\mu_{0} \in \mathcal{P}(\Gamma)$. Indeed, for each $t>0$ and $\theta \in \Theta$, cf (2.1), we have that $\theta \psi_{t} \in \Theta$, and hence $B_{\mu_{0}}\left(\theta \psi_{t}\right)$ is the Bogoliubov functional of a certain state. ${ }^{1}$ The same is true for the left-hand side of (2.23), and the state $\mu_{t}$ contained therein can be condidered as a weak solution of the corresponding Fokker-Planck equation (1.5).

If the initial state is Poissonian with density $\varrho_{0}(x)$, by (2.23) the state $\mu_{t}$ is also Poissonian with the density

$$
\varrho_{t}(x)=\psi_{t}(x) \varrho_{0}(x)+\phi_{t}(x) .
$$

If $m(x) \geq m_{*}>0$ for some $m_{*}$ and all $x \in \mathbb{R}^{d}$, then the solution in (2.21) lies in $\mathcal{K}_{\vartheta_{*}}$ for all $t>0$. Here

$$
\vartheta_{*}=\max \left\{\vartheta_{0} ; \log \left(\|b\| / m_{*}\right)\right\}
$$

Otherwise, the solution in (2.21) is unbounded in $t$. If, for some compact $\Lambda, m(x)=0$ for $x \in \Lambda$, then by (2.21) and (2.22) we get

$$
k_{t}^{(1)}(x)=k_{\mu_{0}}^{(1)}(x)+b(x) t, \quad x \in \Lambda,
$$

\footnotetext{
1 It is an independent thinning of $\mu_{0}$.
} 
that by (1.2), (2.15) and (2.16) yields

$$
\begin{aligned}
\mu_{t}\left(N_{\Lambda}\right) & =\int_{\Gamma}\left|\gamma_{\Lambda}\right| \mu_{t}(d \gamma)=\int_{\Gamma}\left(\sum_{x \in \gamma} I_{\Lambda}(x)\right) \mu_{t}(d \gamma) \\
& =\int_{\Gamma}\left(K I_{\Lambda}\right)(\gamma) \mu_{t}(d \gamma)=\int_{\Lambda} k_{t}^{(1)}(x) d x=\mu_{0}\left(N_{\Lambda}\right)+t \int_{\Lambda} b(x) d x
\end{aligned}
$$

where $I_{\Lambda}$ is the indicator of $\Lambda$. Then $\mu_{t}\left(N_{\Lambda}\right) \rightarrow+\infty$ as $t \rightarrow+\infty$ if $b$ is not identically zero on $\Lambda$.

\subsection{The statements}

For each $\vartheta \in \mathbb{R}$ and $\vartheta^{\prime}>\vartheta$, the expressions in (2.9) and (2.10) can be used to define the corresponding bounded linear operators $L_{\vartheta^{\prime} \vartheta}^{\Delta}$ acting from $\mathcal{K}_{\vartheta}$ to $\mathcal{K}_{\vartheta^{\prime}}$. Their operator norms can be estimated similarly as in [7, eqs. (3.11), (3.13)], which yields, cf. (1.7),

$$
\left\|L_{\vartheta^{\prime} \vartheta}^{\Delta}\right\| \leq \frac{4\|a\|}{e^{2}\left(\vartheta^{\prime}-\vartheta\right)^{2}}+\frac{\|b\| e^{-\vartheta}+\|m\|+\langle a\rangle e^{\vartheta^{\prime}}}{e\left(\vartheta^{\prime}-\vartheta\right)} .
$$

By means of the collection $\left\{L_{\vartheta^{\prime} \vartheta}^{\Delta}\right\}$ with all $\vartheta \in \mathbb{R}$ and $\vartheta^{\prime}>\vartheta$ we introduce a continuous linear operator acting on $\mathcal{K}$, denoted also as $L^{\Delta}$, and thus define the corresponding Cauchy problem (2.8) in this space. By its (global in time) solution we will mean a continuously differentiable function $[0,+\infty) \ni t \mapsto k_{t} \in \mathcal{K}$ such that both equalities in (2.8) hold. Our results are given in the following statements, both based on Assumption 1.1.

Theorem 2.4 (Existence of evolution) For each $\mu_{0} \in \mathcal{P}_{\exp }(\Gamma)$, the problem in (2.8) with $L^{\Delta}: \mathcal{K} \rightarrow \mathcal{K}$ as in (2.9), (2.10) and (2.26) has a unique solution which lies in $\mathcal{K}^{\star}$ and is such that $k_{t}(\emptyset)=1$ for all $t>0$. Therefore, for each $t>0$, there exists a unique state $\mu_{t} \in \mathcal{P}_{\exp }(\Gamma)$ such that $k_{t}=k_{\mu_{t}}$. Moreover, for all $t>0$, the following holds

$$
0 \leq k_{t}(\eta) \leq \sum_{\xi \subset \eta} e\left(\xi ; \phi_{t}\right) e\left(\eta \backslash \xi ; \psi_{t}\right) k_{\mu_{0}}(\eta \backslash \xi),
$$

where $\phi_{t}$ and $\psi_{t}$ are as in (2.22). If the intrinsic mortality rate satisfies $m(x) \geq m_{*}>0$ for all $x \in \mathbb{R}^{d}$, then for all $t>0$ the solution $k_{t}$ lies in $\mathcal{K}_{\vartheta_{*}}$ with $\vartheta_{*}$ given in (2.24).

Theorem 2.5 (Global boundedness) The states $\mu_{t}, t \geq 0$, mentioned in Theorem 2.4 have the property: for every $n \in \mathbb{N}$ and compact $\Lambda \subset \mathbb{R}^{d}$, the following holds

$$
\forall t>0 \quad \mu_{t}\left(N_{\Lambda}^{n}\right) \leq C_{\Lambda}^{(n)}
$$

with some $C_{\Lambda}^{(n)}>0$. If $\mu_{0}$ is such that each $k_{\mu_{0}}^{(n)}$ is a continuous function, then so is $k_{\mu_{t}}^{(n)}$ for all $n \in \mathbb{N}$ and $t>0$. Moreover, $k_{\mu_{t}}^{(1)}$ and $k_{\mu_{t}}^{(2)}$ have the properties as in (1.8). 


\subsection{Comments and comparison}

By (2.25) it follows that the global in time boundedness in the Surgailis model is possible only if $m(x) \geq m_{*}>0$ for all $x \in \mathbb{R}^{d}$. As follows from our Theorem 2.5, adding competition to the Surgailis model with the zero intrinsic mortality rate yields the global in time boundedness. In this case, the competition rate $a(0)$ appears to be an effective mortality, see (4.19) below and the comments following the proof of Theorem 2.5. Note also that the global boundedness as in Theorem 2.5 does not mean that the evolution $k_{\mu_{0}} \mapsto k_{t}$ holds in one and the same $\mathcal{K}_{\vartheta}$ with sufficiently large $\vartheta$. It does if $m(x) \geq m_{*}>0$. Since Theorem 2.4 covers also the case $a \equiv 0$, the solution in (2.21) is unique in the same sense. A partial result on the global boundedness in the model discussed here was obtained in [3, Theorem 1]. Therein, under quite a strong condition imposed on the competition kernel $a$ (which, in particular, implies that it has infinite range), and under the assumption that the evolution of states $\mu_{0} \mapsto \mu_{t}$ exists, there was proved the fact which in the present notations can be formulated as $\mu_{t}\left(N_{\Lambda}\right) \leq C_{\Lambda}$

\section{The existence of the evolution of states}

We follow the line of arguments used in proving Theorem 3.3 in [7] and perform the following three steps:

(i) Defining the Cauchy problem (2.8) with $k_{\mu_{0}} \in \mathcal{K}_{\vartheta_{0}}$ in a given Banach space $\mathcal{K}_{\vartheta}$ with $\vartheta>\vartheta_{0}$, see (2.12) and (2.14), and then showing that this problem has a unique solution $k_{t} \in \mathcal{K}_{\vartheta}$ on a bounded time interval $\left[0, T\left(\vartheta, \vartheta_{0}\right)\right.$ ) (Sect. 3.1).

(ii) Proving that the mentioned solution $k_{t}$ has the properties (a) and (b) in (2.18) ((c) follows by the fact that $\left.k_{t} \in \mathcal{K}_{\vartheta}\right)$. Then $k_{t} \in \mathcal{K}_{\vartheta}^{\star}$ and hence also in $\mathcal{K}_{\vartheta}^{+}$, see (2.20) and (2.19). By Proposition 2.3 it follows that $k_{t}$ is the correlation function of a unique state $\mu_{t}$ (Sect. 3.2).

(iii) Constructing a continuation of $k_{t}$ from $\left[0, T\left(\vartheta, \vartheta_{0}\right)\right)$ to all $t>0$ by means of the fact that $k_{t} \in \mathcal{K}_{\vartheta}^{+}$(Sect. 3.3).

\subsection{Solving the Cauchy problem}

We begin by rewriting $L^{\Delta}$ [given in (2.9), (2.10)] in the following form

$$
\begin{aligned}
L^{\Delta} & =A+B \\
(A k)(\eta) & =-E(\eta) k(\eta), \\
(B k)(\eta) & =-\int_{\mathbb{R}^{d}}\left(\sum_{y \in \eta} a(x-y)\right) k(\eta \cup x) d x+\sum_{x \in \eta} b(x) k(\eta \backslash x) .
\end{aligned}
$$

For $\vartheta \in \mathbb{R}$ and $\vartheta^{\prime}>\vartheta$, let $\mathcal{L}\left(\mathcal{K}_{\vartheta}, \mathcal{K}_{\vartheta^{\prime}}\right)$ be the Banach space of all bounded linear operators acting from $\mathcal{K}_{\vartheta}$ to $\mathcal{K}_{\vartheta^{\prime}}$. Like in (2.26) we define $A_{\vartheta^{\prime} \vartheta}, B_{\vartheta^{\prime} \vartheta} \in \mathcal{L}\left(\mathcal{K}_{\vartheta}, \mathcal{K}_{\vartheta^{\prime}}\right)$, satisfying 


$$
\left\|A_{\vartheta^{\prime} \vartheta}\right\| \leq \frac{4\|a\|}{e^{2}\left(\vartheta^{\prime}-\vartheta\right)^{2}}+\frac{\|m\|}{e\left(\vartheta^{\prime}-\vartheta\right)}, \quad\left\|B_{\vartheta^{\prime} \vartheta}\right\| \leq \frac{\|b\| e^{-\vartheta}+\langle a\rangle e^{\vartheta^{\prime}}}{e\left(\vartheta^{\prime}-\vartheta\right)} .
$$

Now we set, see (2.11),

$$
(S(t) k)(\eta)=\exp (-t E(\eta)) k(\eta), \quad t \geq 0
$$

and then introduce the corresponding $S_{\vartheta^{\prime} \vartheta}(t) \in \mathcal{L}\left(\mathcal{K}_{\vartheta}, \mathcal{K}_{\vartheta^{\prime}}\right), t \geq 0$. By the first estimate in (3.2) one shows that the map

$$
[0,+\infty) \ni t \mapsto S_{\vartheta^{\prime} \vartheta}(t) \in \mathcal{L}\left(\mathcal{K}_{\vartheta}, \mathcal{K}_{\vartheta^{\prime}}\right)
$$

is continuous and such that

$$
\frac{d}{d t} S_{\vartheta^{\prime} \vartheta}(t)=A_{\vartheta^{\prime} \vartheta^{\prime \prime}} S_{\vartheta^{\prime \prime} \vartheta}(t), \quad t>0
$$

holding for each $\vartheta^{\prime \prime} \in\left(\vartheta, \vartheta^{\prime}\right)$. Note that (3.3) may be used to define a bounded multiplication operator, $S_{\vartheta}(t) \in \mathcal{L}\left(\mathcal{K}_{\vartheta}\right):=\mathcal{L}\left(\mathcal{K}_{\vartheta}, \mathcal{K}_{\vartheta}\right)$. However, in this case the map $[0,+\infty) \ni t \mapsto S_{\vartheta}(t) \in \mathcal{L}\left(\mathcal{K}_{\vartheta}\right)$ would not be continuous.

For $\vartheta$ and $\vartheta^{\prime}>\vartheta$ as above, we fix some $\delta<\vartheta^{\prime}-\vartheta$. Then, for a given $l \in \mathbb{N}$, we divide the interval $\left[\vartheta, \vartheta^{\prime}\right]$ into subintervals with endpoints $\vartheta^{s}, s=0,1, \ldots, 2 l+1$, as follows. Set $\vartheta^{0}=\vartheta, \vartheta^{2 l+1}=\vartheta^{\prime}$, and

$$
\begin{array}{rlrl}
\vartheta^{2 s} & =\vartheta+\frac{s}{l+1} \delta+s \epsilon, & \epsilon:=\left(\vartheta^{\prime}-\vartheta-\delta\right) / l, \\
\vartheta^{2 s+1} & =\vartheta+\frac{s+1}{l+1} \delta+s \epsilon, & s & =0,1, \ldots, l .
\end{array}
$$

Then, for $t>0$ and

$$
\left(t, t_{1}, \ldots, t_{l}\right) \in \mathcal{T}_{l}:=\left\{\left(t, t_{1}, \ldots, t_{l}\right): 0 \leq t_{l} \leq t_{l-1} \cdots \leq t_{1} \leq t\right\} \subset \mathbb{R}^{l+1},
$$

define

$$
\begin{aligned}
\Pi_{\vartheta^{\prime} \vartheta}^{(l)}\left(t, t_{1}, \ldots, t_{l}\right)= & S_{\vartheta^{\prime} \vartheta^{2 l}}\left(t-t_{1}\right) B_{\vartheta^{2 l} \vartheta^{2 l-1}} \cdots S_{\vartheta^{2 s+1} \vartheta^{2 s}}\left(t_{l-s}-t_{l-s+1}\right) \\
& \times B_{\vartheta^{2 s} \vartheta^{2 s-1}} \cdots S_{\vartheta^{3} \vartheta^{2}}\left(t_{l-1}-t_{l}\right) B_{\vartheta^{2} \vartheta^{1}} S_{\vartheta^{1} \vartheta}\left(t_{l}\right) .
\end{aligned}
$$

By (3.5), (3.4) and (3.1) one can prove the next statement, cf [7, Proposition 4.6],

Proposition 3.1 For each $l \in \mathbb{N}$, the operators defined in (3.7) have the properties:

(i) for each $\left(t, t_{1}, \ldots, t_{l}\right) \in \mathcal{T}_{l}, \Pi_{\vartheta^{\prime} \vartheta}^{(l)}\left(t, t_{1}, \ldots, t_{l}\right)$ is in $\mathcal{L}\left(\mathcal{K}_{\vartheta}, \mathcal{K}_{\vartheta^{\prime}}\right)$ and the map

$$
\left(t, t_{1}, \ldots, t_{l}\right) \mapsto \Pi_{\vartheta^{\prime} \vartheta}^{(l)}\left(t, t_{1}, \ldots, t_{l}\right) \in \mathcal{L}\left(\mathcal{K}_{\vartheta}, \mathcal{K}_{\vartheta^{\prime}}\right)
$$

is continuous; 
(ii) for fixed $t_{1}, \ldots, t_{l}$ and each $\varepsilon>0$, the map

$$
\left(t_{1}, t_{1}+\varepsilon\right) \ni t \mapsto \Pi_{\vartheta^{\prime} \vartheta}^{(l)}\left(t, t_{1}, \ldots, t_{l}\right) \in \mathcal{L}\left(\mathcal{K}_{\vartheta}, \mathcal{K}_{\vartheta^{\prime}}\right)
$$

is continuously differentiable and such that, for each $\vartheta^{\prime \prime} \in\left(\vartheta, \vartheta^{\prime}\right)$, the following holds

$$
\frac{d}{d t} \Pi_{\vartheta^{\prime} \vartheta}^{(l)}\left(t, t_{1}, \ldots, t_{l}\right)=A_{\vartheta^{\prime} \vartheta^{\prime \prime}} \Pi_{\vartheta^{\prime \prime} \vartheta}^{(l)}\left(t, t_{1}, \ldots, t_{l}\right)
$$

Define

$$
T\left(\vartheta^{\prime}, \vartheta\right)=\frac{\vartheta^{\prime}-\vartheta}{\|b\| e^{-\vartheta}+\langle a\rangle e^{\vartheta^{\prime}}} .
$$

Then assume that $k_{\mu_{0}} \in \mathcal{K}_{\vartheta_{0}}$, fix some $\vartheta_{1}>\vartheta_{0}$, and set

$$
\Theta=\left\{\left(\vartheta, \vartheta^{\prime}, t\right): \vartheta_{0} \leq \vartheta<\vartheta^{\prime} \leq \vartheta_{1}, \quad t<T\left(\vartheta^{\prime}, \vartheta\right)\right\}
$$

Proposition 3.2 There exists a family of linear operators, $\left\{Q_{\vartheta^{\prime} \vartheta}(t):\left(\vartheta, \vartheta^{\prime}, t\right) \in \Theta\right\}$, each element of which is in the corresponding $\mathcal{L}\left(\mathcal{K}_{\vartheta}, \mathcal{K}_{\vartheta^{\prime}}\right)$ and has the following properties:

(i) the map $\left[0, T\left(\vartheta^{\prime}, \vartheta\right)\right) \ni t \mapsto Q_{\vartheta^{\prime} \vartheta}(t) \in \mathcal{L}\left(\mathcal{K}_{\vartheta}, \mathcal{K}_{\vartheta^{\prime}}\right)$ is continuous and $Q_{\vartheta^{\prime} \vartheta}(0)$ is the embedding $\mathcal{K}_{\vartheta} \hookrightarrow \mathcal{K}_{\vartheta^{\prime}}$;

(ii) for each $\vartheta^{\prime \prime} \in\left(\vartheta, \vartheta^{\prime}\right)$ and $t<T\left(\vartheta^{\prime \prime}, \vartheta\right)$, the following holds

$$
\frac{d}{d t} Q_{\vartheta^{\prime} \vartheta}(t)=L_{\vartheta^{\prime} \vartheta^{\prime \prime}}^{\Delta} Q_{\vartheta^{\prime \prime} \vartheta}(t)
$$

Proof We go along the line of arguments used in the proof of Lemma 4.5 in [7]. Take any $T<T\left(\vartheta^{\prime}, \vartheta\right)$ and then pick $\vartheta^{\prime \prime} \in\left(\vartheta, \vartheta^{\prime}\right]$ and a positive $\delta<\vartheta^{\prime \prime}-\vartheta$ such that also $T<T_{\delta}:=T\left(\vartheta^{\prime \prime}-\delta, \vartheta\right)$. For these values of the parameters, take $\Pi_{\vartheta}^{(l)} \vartheta$ as in (3.7) and then, for $n \in \mathbb{N}$, set

$$
Q_{\vartheta^{\prime \prime} \vartheta}^{(n)}(t)=S_{\vartheta^{\prime \prime} \vartheta}(t)+\sum_{l=1}^{n} \int_{0}^{t} \int_{0}^{t_{1}} \cdots \int_{0}^{t_{l-1}} \Pi_{\vartheta^{\prime \prime} \vartheta}^{(l)}\left(t, t_{1} \ldots, t_{l}\right) d t_{l} \cdots d t_{1}
$$

By (3.3) and the second estimate in (3.2) we have from (3.7) that

$$
\left\|\Pi_{\vartheta^{\prime \prime} \vartheta}^{(l)}\left(t, t_{1}, \ldots, t_{l}\right)\right\| \leq\left(\frac{l}{e T_{\delta}}\right)^{l}
$$

holding for all $l=1, \ldots, n$. By (3.12), for $t \in[0, T)$, this yields

$$
\begin{aligned}
\left\|Q_{\vartheta}^{(n) \vartheta}(t)-Q_{\vartheta}^{(n-1)}(t)\right\| & \leq \int_{0}^{t} \int_{0}^{t_{1}} \cdots \int_{0}^{t_{n-1}}\left\|\Pi_{\vartheta "}^{(n)}\left(t, t_{1} \ldots, t_{n}\right)\right\| d t_{l} \cdots d t_{n} \\
& \leq \frac{1}{n !}\left(\frac{n}{e}\right)^{n}\left(\frac{T}{T_{\delta}}\right)^{n} .
\end{aligned}
$$


Hence, for all $t \in[0, T],\left\{Q_{\vartheta^{\prime \prime} \vartheta}^{(n)}(t)\right\}_{n \in \mathbb{N}}$ is a Cauchy sequence in $\mathcal{L}\left(\mathcal{K}_{\vartheta}, \mathcal{K}_{\vartheta^{\prime \prime}}\right)$. The operator $Q_{\vartheta}{ }^{\prime \prime}(t)$ in question is then its limit. The continuity in (i) follows by the fact that the convergence to $Q_{\vartheta^{\prime \prime} \vartheta}(t)$ is uniform on [0,T]. Moreover, by (3.12) we have that $Q_{\vartheta \vartheta^{\prime \prime} \vartheta}^{(n)}(0)=S_{\vartheta^{\prime \prime} \vartheta}(0)$, cf. (3.3), which yields the stated property of $Q_{\vartheta^{\prime \prime} \vartheta}(0)$. Finally, (3.11) follows from (3.8) by the same arguments.

From (3.11) one can get that the family mentioned in Proposition 3.2 enjoys the following 'semigroup' property

$$
Q_{\vartheta^{\prime} \vartheta}(t+s)=Q_{\vartheta^{\prime} \vartheta^{\prime \prime}}(t) Q_{\vartheta^{\prime \prime} \vartheta}(s)
$$

holding whenever $\left(\vartheta, \vartheta^{\prime}, t+s\right),\left(\vartheta^{\prime \prime}, \vartheta^{\prime}, t\right)$, and $\left(\vartheta, \vartheta^{\prime \prime}, s\right)$ are in $\Theta$.

Now we make precise which Cauchy problem we are going to solve. Set

$$
\mathcal{D}_{\vartheta}=\left\{k \in \mathcal{K}_{\vartheta}: L^{\Delta} k \in \mathcal{K}_{\vartheta}\right\}
$$

where $L^{\Delta}$ is as in (2.9). This defines an unbounded linear operator $L_{\vartheta}^{\Delta}: \mathcal{D}_{\vartheta} \rightarrow \mathcal{K}_{\vartheta}$, being the extension of the operators $L_{\vartheta^{\prime \prime} \vartheta_{0}}^{\Delta}: \mathcal{K}_{\vartheta_{0}} \rightarrow \mathcal{K}_{\vartheta^{\prime \prime}} \hookrightarrow \mathcal{K}_{\vartheta}$ with $\vartheta^{\prime \prime} \in\left(\vartheta_{0}, \vartheta\right)$ and all $\vartheta_{0}<\vartheta$, cf. (2.14). Then we consider the Cauchy problem (2.8) in $\mathcal{K}_{\vartheta_{1}}$ with this operator $L_{\vartheta_{1}}^{\Delta}$ and $k_{\mu_{0}} \in \mathcal{K}_{\vartheta_{0}}$. By its classical solution we understand the corresponding map $t \mapsto k_{t} \in \mathcal{D}_{\vartheta_{1}}$, continuously differentiable in $\mathcal{K}_{\vartheta_{1}}$.

Lemma 3.3 Let $\vartheta_{0}$ and $\vartheta_{1}$ be as in (3.10). Then for each $k_{\mu_{0}} \in \mathcal{K}_{\vartheta_{0}}$, the problem (2.8) as described above, cf. (3.16) below, has a unique solution $k_{t} \in \mathcal{K}_{\vartheta_{1}}$ with $t \in\left[0, T\left(\vartheta_{1}, \vartheta_{0}\right)\right)$ given by the formula

$$
k_{t}=Q_{\vartheta_{1} \vartheta_{0}}(t) k_{\mu_{0}}
$$

such that $k_{t}(\emptyset)=1$ for all $t \in\left[0, T\left(\vartheta_{1}, \vartheta_{0}\right)\right)$.

Proof For each $t<T\left(\vartheta_{1}, \vartheta_{0}\right)$, one finds $\vartheta^{\prime \prime} \in\left(\vartheta_{0}, \vartheta_{1}\right)$ such that also $t<T\left(\vartheta^{\prime \prime}, \vartheta_{0}\right)$. By (3.11) we then get

$$
\frac{d}{d t} k_{t}=L_{\vartheta_{1} \vartheta^{\prime \prime}}^{\Delta} k_{t}=L_{\vartheta_{1}}^{\Delta} k_{t}
$$

By claim (i) of Proposition 3.2 we have that $k_{0}=k_{\mu_{0}}$. Moreover, $k_{t}(\emptyset)=1$ for all $t \in\left[0, T\left(\vartheta_{1}, \vartheta_{0}\right)\right)$ since $k_{0}=k_{\mu_{0}}$, see (b) in (2.18), and

$$
\left(\frac{d}{d t} k_{t}\right)(\emptyset)=\left(L_{\vartheta_{1} \vartheta^{\prime \prime}}^{\Delta} k_{t}\right)(\emptyset)=0,
$$

which follows from (2.10)-(2.9). The stated uniqueness follows by the arguments used in the proof of Lemma 4.8 in [7].

Remark 3.4 As in the proof of Lemma 3.3 one can show that, for each $t \in$ $\left[0, T\left(\vartheta_{1}, \vartheta_{0}\right)\right)$, the following holds:

$$
Q_{\vartheta_{1} \vartheta_{0}}(t): \mathcal{K}_{\vartheta_{0}} \rightarrow \mathcal{D}_{\vartheta_{1}}
$$


see (3.14), and

$$
\frac{d}{d t} Q_{\vartheta_{1} \vartheta_{0}}(t)=L_{\vartheta_{1}}^{\Delta} Q_{\vartheta_{1} \vartheta_{0}}(t)
$$

Now we construct the evolution of functions $G_{0} \mapsto G_{t}$ such that, for $k \in \mathcal{K}_{\vartheta}$, the following holds, cf. (2.16),

$$
\left\langle\left\langle G_{0}, Q_{\vartheta^{\prime} \vartheta}(t) k\right\rangle\right\rangle=\left\langle\left\langle G_{t}, k\right\rangle\right\rangle, \quad t<T\left(\vartheta^{\prime}, \vartheta\right) .
$$

To this end, we introduce, cf. (2.12) and (2.13),

$$
\begin{aligned}
|G|_{\vartheta} & =\int_{\Gamma_{0}}|G(\eta)| \exp (\vartheta|\eta|) \lambda(d \eta), \\
\mathcal{G}_{\vartheta} & =\left\{G: \Gamma_{0} \rightarrow \mathbb{R}:|G|_{\vartheta}<\infty\right\} .
\end{aligned}
$$

Clearly, $\mathcal{G}_{\vartheta^{\prime}} \hookrightarrow \mathcal{G}_{\vartheta}$ for $\vartheta<\vartheta^{\prime}$; hence, we have introduced another scale of Banach spaces, cf. (2.14). As in (3.1) and (3.3), we define the corresponding multiplication operators $A_{\vartheta \vartheta^{\prime}}$ and $S_{\vartheta \vartheta^{\prime}}(t)$, and also $C_{\vartheta \vartheta^{\prime}} \in \mathcal{L}\left(\mathcal{G}_{\vartheta^{\prime}}, \mathcal{G}_{\vartheta}\right)$ which acts as

$$
(C G)(\eta)=-\sum_{x \in \eta}\left(\sum_{y \in \eta \backslash x} a(x-y)\right) G(\eta \backslash x)+\int_{\mathbb{R}^{d}} b(x) G(\eta \cup x) d x,
$$

and thus satisfies, cf. (3.2),

$$
\left\|C_{\vartheta \vartheta^{\prime}}\right\| \leq \frac{\|b\| e^{-\vartheta}+\langle a\rangle e^{\vartheta^{\prime}}}{e\left(\vartheta^{\prime}-\vartheta\right)} .
$$

Now, for the same division of $\left[\vartheta, \vartheta^{\prime}\right]$ as in (3.6), we introduce, $\mathrm{cf}$. (3.7),

$$
\begin{aligned}
\Omega_{\vartheta \vartheta^{\prime}}^{l}\left(t, t_{1}, \ldots, t_{l}\right)= & S_{\vartheta \vartheta^{1}}\left(t_{l}\right) C_{\vartheta^{1} \vartheta^{2}} S_{\vartheta^{2} \vartheta^{3}}\left(t_{l-1}-t_{l}\right) \cdots C_{\vartheta^{2 s-1} \vartheta^{2 s}} \\
& \times S_{\vartheta^{2 s} \vartheta^{2 s+1}}\left(t_{l-s}-t_{l-s+1}\right) \cdots C_{\vartheta^{2 l-1} \vartheta^{2 l}} S_{\vartheta^{2 l} \vartheta^{\prime}}\left(t-t_{1}\right) .
\end{aligned}
$$

For this $\Omega_{\vartheta \vartheta^{\prime}}^{l}$, one can get the properties analogous to those stated in Proposition 3.1. Next, for $n \in \mathbb{N}$, we define, cf. (3.12),

$$
H_{\vartheta \vartheta^{\prime}}^{(n)}(t)=S_{\vartheta \vartheta^{\prime}}(t)+\sum_{l=1}^{n} \int_{0}^{t} \int_{0}^{t_{1}} \cdots \int_{0}^{t_{l-1}} \Omega_{\vartheta \vartheta^{\prime}}^{l}\left(t, t_{1}, \ldots, t_{l}\right) d t_{l} \cdots d t_{1} .
$$

As in the proof of Proposition 3.2, by means of (3.19) we then show that the sequence $\left\{H_{\vartheta \vartheta^{\prime}}^{(n)}(t)\right\}_{n \in \mathbb{N}}$ converges in $\mathcal{L}\left(\mathcal{G}_{\vartheta^{\prime}}, \mathcal{G}_{\vartheta}\right)$, uniformly on compact subsets of $\left[0, T\left(\vartheta^{\prime}, \vartheta\right)\right)$. Let $H_{\vartheta \vartheta}(t)$ be the limit. Then, by the very construction in (3.20), it follows that, cf. (3.17),

$$
\left\langle\left\langle H_{\vartheta \vartheta^{\prime}}(t) G, k\right\rangle\right\rangle=\left\langle\left\langle G, Q_{\vartheta^{\prime} \vartheta}(t) k\right\rangle, \quad t \in\left[0, T\left(\vartheta^{\prime}, \vartheta\right)\right),\right.
$$

holding for each $G \in \mathcal{G}_{\vartheta^{\prime}}$ and $k \in \mathcal{K}_{\vartheta}$. 


\subsection{The identification}

Our next step is based on the following statement.

Lemma 3.5 Let $\left\{Q_{\vartheta^{\prime} \vartheta}(t):\left(\vartheta, \vartheta^{\prime}, t\right) \in \Theta\right\}$ be the family as in Proposition 3.2. Then, for each $\vartheta$ and $\vartheta^{\prime}$ and $t \in\left[0, T\left(\vartheta^{\prime}, \vartheta\right) / 2\right)$, we have that $Q_{\vartheta^{\prime} \vartheta}(t): \mathcal{K}_{\vartheta}^{\star} \rightarrow \mathcal{K}_{\vartheta^{\prime}}^{\star}$.

We prove this lemma in a number of steps. First we introduce auxiliary models, indexed by $\sigma>0$, for which we construct the families of operators $Q_{\vartheta^{\prime} \vartheta^{\prime}}^{\sigma}(t)$ similar as in Proposition 3.2. Then we prove that these families have the property stated in Lemma 3.5. Thereafter, we show that

$$
\left\langle\left\langle G, Q_{\vartheta_{1} \vartheta_{0}}^{\sigma}(t) k_{0}\right\rangle\right\rangle=:\left\langle\left\langle G, k_{t}^{\sigma}\right\rangle\right\rangle \rightarrow\left\langle\left\langle G, k_{t}\right\rangle\right\rangle, \quad \text { as } \sigma \rightarrow 0
$$

holding for each $G \in B_{\mathrm{bs}}^{\star}\left(\Gamma_{0}\right)$ and $k_{t}$ as in Lemma 3.3 with $t \in\left[0, T\left(\vartheta^{\prime}, \vartheta\right) / 2\right)$, see (2.17). By Proposition 2.3 this yields the fact we wish to prove.

\subsubsection{Auxiliary models}

For $\sigma>0$, we set

$$
\varphi_{\sigma}(x)=\exp \left(-\sigma|x|^{2}\right), \quad b_{\sigma}(x)=b(x) \varphi_{\sigma}(x)
$$

Let also $L^{\Delta, \sigma}$ stand for $L^{\Delta}$ as in (2.9) with $b$ replaced by $b_{\sigma}$. Note that $\left\|b_{\sigma}\right\| \leq$ $\|b\|$. Clearly, for this $L^{\Delta, \sigma}$, we can perform the same construction as in the previous subsection and obtain the family $\left\{Q_{\vartheta^{\prime} \vartheta}^{\sigma}(t):\left(\vartheta, \vartheta^{\prime}, t\right) \in \Theta\right\}$ as in Proposition 3.2 with $\Theta$ and $T\left(\vartheta^{\prime}, \vartheta\right)$ given in (3.10) and (3.9), respectively. Note also that $Q_{\vartheta^{\prime} \vartheta}^{\sigma}(t)$ satisfy, cf. (3.11) and Remark 3.4,

$$
\frac{d}{d t} Q_{\vartheta^{\prime} \vartheta}^{\sigma}(t)=L_{\vartheta^{\prime} \vartheta^{\prime \prime}}^{\Delta, \sigma} Q_{\vartheta^{\prime \prime} \vartheta}^{\sigma}(t)=L_{\vartheta^{\prime}}^{\Delta, \sigma} Q_{\vartheta^{\prime} \vartheta}^{\sigma}(t)
$$

Like in (3.15) we then set

$$
k_{t}^{\sigma}=Q_{\vartheta_{1} \vartheta_{0}}^{\sigma}(t) k_{\mu_{0}}, \quad t<T\left(\vartheta_{1}, \vartheta_{0}\right)
$$

Also as above, we construct the operators $H_{\vartheta \vartheta^{\prime}}^{\sigma}(t)$ such that, cf. (3.21),

$$
\left\langle\left\langle H_{\vartheta \vartheta^{\prime}}^{\sigma}(t) G, k\right\rangle\right\rangle=\left\langle\left\langle G, Q_{\vartheta^{\prime} \vartheta}^{\sigma}(t) k\right\rangle\right\rangle
$$

holding for appropriate $G$ and $k$.

Proposition 3.6 Assume that $Q_{\vartheta_{1} \vartheta_{0}}^{\sigma}: \mathcal{K}_{\vartheta_{0}}^{\star} \rightarrow \mathcal{K}_{\vartheta_{1}}^{\star}$ for all $t<T\left(\vartheta_{1}, \vartheta_{0}\right)$. Then, for all $t<T\left(\vartheta_{1}, \vartheta_{0}\right) / 2$ and $G \in B_{\mathrm{bs}}\left(\Gamma_{0}\right)$, the convergence in (3.22) holds. 
Proof Take $\vartheta=\left(\vartheta_{1}+\vartheta_{0}\right) / 2$ and then pick $\vartheta^{\prime} \in\left(\vartheta, \vartheta_{1}\right)$ such that

$$
\widetilde{T}:=\frac{1}{2} T\left(\vartheta_{1}, \vartheta_{0}\right) \leq \min \left\{T\left(\vartheta_{1}, \vartheta^{\prime}\right) ; T\left(\vartheta, \vartheta_{0}\right)\right\}
$$

which is possible in view of the continuous dependence of $T\left(\vartheta^{\prime}, \vartheta\right)$ on both its arguments, see (3.9). For $t<T\left(\vartheta_{1}, \vartheta_{0}\right) / 2$, by (3.15) and (3.25) we get that

$$
k_{t}-k_{t}^{\sigma}=\int_{0}^{t} Q_{\vartheta_{1} \vartheta^{\prime}}(t-s)\left(L_{\vartheta^{\prime} \vartheta}^{\Delta}-L_{\vartheta^{\prime} \vartheta}^{\Delta, \sigma}\right) k_{s}^{\sigma} d s=: \int_{0}^{t} Q_{\vartheta_{1} \vartheta^{\prime}}(t-s) D_{\vartheta^{\prime} \vartheta} k_{s}^{\sigma} d s,
$$

where (2.9),

$$
(D k)(\eta)=\sum_{x \in \eta}\left(1-\varphi_{\sigma}(x)\right) b(x) k(\eta \backslash x),
$$

see (2.9) and $k_{s}^{\sigma}$ lies in $\mathcal{K}_{\vartheta}$, which is possible since

$$
s \leq t<\frac{1}{2} T\left(\vartheta_{1}, \vartheta_{0}\right) \leq T\left(\vartheta, \vartheta_{0}\right)
$$

see (3.27). Take $G \in B_{\mathrm{bs}}$. Since it lies in each $\mathcal{G}_{\vartheta}$, and hence in $\mathcal{G}_{\vartheta_{1}}$, we can get

$$
H_{\vartheta^{\prime} \vartheta_{1}}(t-s) G=: G_{t-s} \in \mathcal{G}_{\vartheta^{\prime}}, \quad t-s<T\left(\vartheta_{1}, \vartheta_{0}\right) / 2
$$

see (3.27). For this $G$, by (3.21) and (3.28) we have

$$
\begin{aligned}
& \psi_{\sigma}(t):=\left\langle\left\langle G, k_{t}-k_{t}^{\sigma}\right\rangle\right\rangle=\int_{0}^{t}\left\langle\left\langle G_{t-s}, D_{\vartheta^{\prime} \vartheta} k_{s}^{\sigma}\right\rangle\right\rangle d s \\
& =\int_{0}^{t}\left(\int_{\Gamma_{0}} \int_{\mathbb{R}^{d}} \frac{1}{|\eta|+1} G_{t-s}(\eta \cup x) b(x)\left(1-\varphi_{\sigma}(x)\right)(|\eta|+1) k_{s}^{\sigma}(\eta) d x \lambda(d \eta)\right) d s .
\end{aligned}
$$

To get the latter line we also used (3.29). Recall that here $G_{t-s} \in \mathcal{G}_{\vartheta^{\prime}}$ and $k_{s}^{\sigma} \in \mathcal{K}_{\vartheta}$ with $\vartheta<\vartheta^{\prime}$. Let us prove that

$$
g_{s}(x):=\int_{\Gamma_{0}} \frac{1}{|\eta|+1}\left|G_{s}(\eta \cup x)\right| \exp \left(\vartheta^{\prime}|\eta|\right) \lambda(d \eta)
$$

lies in $L^{1}\left(\mathbb{R}^{d}\right)$ for each $s \leq \widetilde{T}$. Indeed, by (2.5) and (3.18) we have

$$
\left\|g_{S}\right\|_{L^{1}\left(\mathbb{R}^{d}\right)} \leq e^{-\vartheta^{\prime}} \sup _{s \in[0, \widetilde{T}]}\left|G_{S}\right|_{\vartheta^{\prime}}
$$


We use this in (3.30) to get

$$
\begin{aligned}
\left|\psi_{\sigma}(t)\right| & \leq \sup _{s \in[0, \widetilde{T}]}\left\|k_{s}\right\|_{\vartheta} \frac{\|b\| e^{\vartheta^{\prime}-\vartheta-1}}{\vartheta^{\prime}-\vartheta} \int_{0}^{t} \int_{\mathbb{R}^{d}} g_{s}(x)\left(1-\varphi_{\sigma}(x)\right) d x d s \rightarrow 0, \\
\text { as } \sigma & \rightarrow 0 .
\end{aligned}
$$

The latter convergence follows by (3.31) and the Lebesgue dominated convergence theorem. This completes the proof.

\subsubsection{Auxiliary evolutions}

Now we turn to proving that the assumption of Proposition 3.6 holds true. For a compact $\Lambda$, by $\Gamma_{\Lambda}$ we denote the set of configurations $\eta$ contained in $\Lambda$. It is a measurable subset of $\Gamma_{0}$, i.e., $\Gamma_{\Lambda} \in \mathcal{B}(\Gamma)$. Recall that $\mathcal{B}(\Gamma)$ can be generated by the cylinder sets $\Gamma^{\Lambda, n}$ with all possible compact $\Lambda$ and $n \in \mathbb{N}_{0}$. Let $\mathcal{B}\left(\Gamma_{\Lambda}\right)$ denote the sub- $\sigma$-field of $\mathcal{B}(\Gamma)$ consisting of $A \subset \Gamma_{\Lambda}$. For $A \in \mathcal{B}\left(\Gamma_{\Lambda}\right)$, we set $C_{\Lambda}(A)=\left\{\gamma \in \Gamma: \gamma_{\Lambda} \in A\right\}$. Then, for a state $\mu$, we define $\mu^{\Lambda}$ by setting $\mu^{\Lambda}(A)=\mu\left(C_{\Lambda}(A)\right)$; thereby, $\mu^{\Lambda}$ is a probability measure on $\mathcal{B}\left(\Gamma_{\Lambda}\right)$. It is possible to show, see [8], that for each compact $\Lambda$ and $\mu \in \mathcal{P}_{\exp }(\Gamma)$, the measure $\mu^{\Lambda}$ has density with respect to the Lebesgue-Poisson measure defined in (2.5), which we denote by $R_{\mu}^{\Lambda}$. Moreover, the correlation function $k_{\mu}$ and the density $R_{\mu}^{\Lambda}$ satisfy

$$
k_{\mu}(\eta)=\int_{\Gamma_{\Lambda}} R_{\mu}^{\Lambda}(\eta \cup \xi) \lambda(d \xi), \quad \eta \in \Gamma_{\Lambda} .
$$

Let $\mu_{0} \in \mathcal{P}_{\exp }(\Gamma)$ be the initial state as in Lemma 3.3. Fix some compact $\Lambda$ and $N \in \mathbb{N}$, and then, for $\eta \in \Gamma_{0}$, set

$$
R_{0}^{\Lambda, N}(\eta)= \begin{cases}R_{\mu_{0}}^{\Lambda}(\eta), & \text { if } \eta \subset \Lambda \text { and }|\eta| \leq N \\ 0, & \text { otherwise. }\end{cases}
$$

Clearly, $R_{0}^{\Lambda, N} \in \mathcal{G}_{\vartheta}$ with any $\vartheta \in \mathbb{R}$, and $R_{0}^{\Lambda, N}(\eta) \geq 0$ for $\lambda$-almost all $\eta \in \Gamma_{0}$.

Let us now consider the auxiliary model specified by $L^{\Delta, \sigma}$, and also by $L^{\sigma}$ which one obtains by replacing in (1.6) $b$ by $b_{\sigma}$, see (3.23). Then the equation for the densities obtained from the Fokker-Planck equation (1.5) takes the form

$$
\begin{aligned}
& \frac{d}{d t} R_{t}(\eta)=\left(L^{\dagger} R_{t}\right)(\eta) \\
& :=-\Psi_{\sigma}(\eta) R_{t}(\eta)+\sum_{x \in \eta} b_{\sigma}(x) R_{t}(\eta \backslash x) \\
& \quad+\int_{\mathbb{R}^{d}}\left(\sum_{y \in \eta} a(x-y)\right) R_{t}(\eta \cup x) d x,
\end{aligned}
$$


where

$$
\Psi_{\sigma}(\eta):=E(\eta)+\left\langle b_{\sigma}\right\rangle, \quad\left\langle b_{\sigma}\right\rangle:=\int_{\mathbb{R}^{d}} b(x) \varphi_{\sigma}(x) d x .
$$

Set

$$
\mathcal{G}_{\vartheta}^{+}=\left\{G \in \mathcal{G}_{\vartheta}: G(\eta) \geq 0, \text { for } \lambda-\text { a.a. } \eta \in \Gamma_{0}\right\}
$$

and also

$$
\mathcal{D}=\left\{R \in \mathcal{G}_{0}: \Psi_{\sigma} R \in \mathcal{G}_{0}\right\}, \quad \mathcal{D}^{+}=\mathcal{D} \bigcap \mathcal{G}_{0}^{+}
$$

Proposition 3.7 The operator $\left(L^{\dagger}, \mathcal{D}\right)$ defined in (3.34) and (3.36) is the generator of a substochastic semigroup $S^{\dagger}=\left\{S^{\dagger}(t)\right\}_{t \geq 0}$ on $\mathcal{G}_{0}$, which leaves invariant each $\mathcal{G}_{\vartheta}$, $\vartheta>0$.

Proof In this statement we mean that

$$
\begin{array}{ll}
\forall t \geq 0 \quad \text { (a) } S^{\dagger}(t): \mathcal{G}_{0}^{+} \rightarrow \mathcal{D}^{+} ; \\
\\
\text {(b) }\left|S^{\dagger}(t) R\right|_{0} \leq 1, \text { whenever }|R|_{0} \leq 1 \text { and } R \in \mathcal{G}_{0}^{+} ; \\
\text {(c) } S^{\dagger}(t): \mathcal{G}_{\vartheta}^{+} \rightarrow \mathcal{G}_{\vartheta}^{+} \text {, for all } \vartheta>0 .
\end{array}
$$

We use the Thieme-Voigt theorem in the form of [10, Propositions 3.1 and 3.2]. By this theorem the proof amounts to checking the validity of the following inequalities:

$$
\begin{aligned}
& \forall R \in \mathcal{D}^{+} \quad \int_{\Gamma_{0}}\left(L^{\dagger} R\right)(\eta) \lambda(d \eta) \leq 0, \\
& \forall \vartheta>0 \quad\left(L^{\sigma} G_{\vartheta}\right)(\eta)+\varepsilon \Psi_{\sigma}(\eta) \leq C G_{\vartheta}(\eta), \quad G_{\vartheta}(\eta):=e^{\vartheta|\eta|},
\end{aligned}
$$

holding for some positive $C$ and $\varepsilon$. Recall that $\Psi_{\sigma}$ is defined in (3.35). By direct inspection we get from (3.34) that the left-hand side of the first line in (3.38) equals zero for each $R \in \mathcal{D}$. Proving the second inequality in (3.38) reduces to showing that, for each $\vartheta>0$, the function

$$
\Sigma(\eta):=-E(\eta)\left(1-e^{-\vartheta}\right)+\left\langle b_{\sigma}\right\rangle\left(e^{\vartheta}-1\right)+\varepsilon \Psi_{\sigma}(\eta) e^{-\vartheta|\eta|}, \quad \eta \in \Gamma_{0},
$$

is bounded from above, which is obviously the case.

The second auxiliary evolution is supposed to be constructed in $\mathcal{G}_{\vartheta}$. It is generated by the operator $\widehat{L}_{\vartheta}$ the action of which coincides with that of $L^{\Delta, \sigma}$, see (2.9) and (2.10) with $b$ replaced by $b_{\sigma}$. The domain of this operator is

$$
\widehat{\mathcal{D}}_{\vartheta}=\left\{q \in \mathcal{G}_{\vartheta}: \Psi_{\sigma}(\cdot) q \in \mathcal{G}_{\vartheta}\right\} .
$$

Proposition 3.8 For each $\vartheta>0$, the operator $\left(\widehat{L}_{\vartheta}, \widehat{\mathcal{D}}_{\vartheta}\right)$ is the generator of a $C_{0^{-}}$ semigroup $\widehat{S}_{\vartheta}:=\left\{\widehat{S}_{\vartheta}(t)\right\}_{t \geq 0}$ of bounded operators on $\mathcal{G}_{\vartheta}$.

Proof As in the proof of Lemma 5.5 in [7], we pass from $q$ to $w$ by setting $w(\eta)=$ $(-1)^{|\eta|} q(\eta)$, and hence to $\widetilde{L}_{\vartheta}$ defined on the same domain (3.39) by the relation 
$\left(\widetilde{L}_{\vartheta} w\right)(\eta)=(-1)^{|\eta|}\left(\widehat{L}_{\vartheta} q\right)(\eta)$. Then we just prove that $\left(\widetilde{L}_{\vartheta}, \widehat{\mathcal{D}}_{\vartheta}\right)$ generates a $C_{0^{-}}$ semigroup on $\mathcal{G}_{\vartheta}$. In view of $(2.10)-(2.9)$, we have

$$
\begin{aligned}
\widetilde{L}_{\vartheta} & =\widetilde{A}+\widetilde{B}+\widetilde{C} \\
(\widetilde{A} w)(\eta) & =-E(\eta) w(\eta), \quad(\widetilde{B} w)(\eta)=\int_{\mathbb{R}^{d}}\left(\sum_{y \in \eta} a(x-y)\right) w(\eta \cup x) d x, \\
(\widetilde{C} w)(\eta) & =-\sum_{x \in \eta} b_{\sigma}(x) w(\eta \backslash x) .
\end{aligned}
$$

By (3.18) we get

$$
|\widetilde{C} w|_{\vartheta} \leq e^{\vartheta}\left\langle b_{\sigma}\right\rangle|w|_{\vartheta}
$$

hence $\widetilde{C}$ is a bounded operator. For $w \in \mathcal{G}_{\vartheta}^{+}$, we have

$$
\begin{aligned}
|\widetilde{B} w|_{\vartheta} & =\int_{\Gamma_{0}} e^{\vartheta|\eta|}\left(\int_{\mathbb{R}^{d}}\left(\sum_{y \in \eta} a(x-y)\right) w(\eta \cup x) d x\right) \lambda(d \eta) \\
& =\int_{\Gamma_{0}} e^{\vartheta(|\eta|-1)}\left(\sum_{x \in \eta} \sum_{y \in \eta \backslash x} a(x-y)\right) w(\eta) \lambda(d \eta) \\
& \leq e^{-\vartheta} \int_{\Gamma_{0}} e^{\vartheta|\eta|} E(\eta) w(\eta) \lambda(d \eta)=e^{-\vartheta}|\widetilde{A} w|_{\vartheta}<|\widetilde{A} w|_{\vartheta} .
\end{aligned}
$$

The latter estimate allows us to apply here the Thieme-Voigt theorem, see [10, Proposition 3.1] by which $\widetilde{A}+\widetilde{B}$ generates a substochastic semigroup in $\mathcal{G}_{\vartheta}$. Thus, $\widetilde{L}_{\vartheta}$ generates a $C_{0}$-semigroup since $\widetilde{C}$ is bounded. This completes the proof.

Now for $R_{0}^{\Lambda, N}$ defined in (3.33), we set

$$
q_{0}^{\Lambda, N}(\eta)=\int_{\Gamma_{0}} R_{0}^{\Lambda, N}(\eta \cup \xi) \lambda(d \xi), \quad \eta \in \Gamma_{0} .
$$

By (3.32)

$$
0 \leq q_{0}^{\Lambda, N}(\eta) \leq k_{\mu_{0}}(\eta)
$$

Hence, $q_{0}^{\Lambda, N} \in \mathcal{K}_{\vartheta_{0}}$. By (3.33) $R_{0}^{\Lambda, N}$ lies in each $\mathcal{G}_{\vartheta}, \vartheta \geq 0$. At the same time,

$$
\begin{aligned}
\left|q_{0}^{\Lambda, N}\right|_{\vartheta} & =\int_{\Gamma_{0}} \int_{\Gamma_{0}} e^{\vartheta|\eta|} R_{0}^{\Lambda, N}(\eta \cup \xi) \lambda(d \eta) \lambda(d \xi) \\
& =\int_{\Gamma_{0}}\left(\sum_{\eta \subset \xi} e^{\vartheta|\eta|}\right) R_{0}^{\Lambda, N}(\xi) \lambda(d \xi)=\left|R_{0}^{\Lambda, N}\right|_{\beta},
\end{aligned}
$$


where $\beta>0$ is to satisfy $e^{\beta}=1+e^{\vartheta}$. Hence, $q_{0}^{\Lambda, N} \in \mathcal{G}_{\vartheta}$ for each $\vartheta>0$. In view of this, $q_{0}^{\Lambda, N} \in \widehat{\mathcal{D}}_{\vartheta}$ for each $\vartheta>0$, see (3.39). Consider the problem in $\mathcal{G}_{\vartheta}$

$$
\frac{d}{d t} q_{t}=\widehat{L}_{\vartheta} q_{t},\left.\quad q_{t}\right|_{t=0}=q_{0}^{\Lambda, N}
$$

Proposition 3.9 For each $\vartheta>0$, the problem in (3.42) has a unique global solution $q_{t} \in \widehat{\mathcal{D}}_{\vartheta}$ such that, for each $G \in B_{\mathrm{bs}}^{\star}\left(\Gamma_{0}\right)$, the following holds

$$
\left\langle\left\langle G, q_{t}\right\rangle\right\rangle \geq 0
$$

Proof By Proposition 3.8 the problem in (3.42) has a unique global solution given by

$$
q_{t}=\widehat{S}_{\vartheta}(t) q_{0}^{\Lambda, N}
$$

On the other hand, this solution can be sought in the form

$$
q_{t}(\eta)=\int_{\Gamma_{0}}\left(S^{\dagger}(t) R_{0}^{\Lambda, N}\right)(\eta \cup \xi) \lambda(d \xi),
$$

where $S^{\dagger}$ is the semigroup constructed in Proposition 3.7. Indeed, by direct inspection one verifies that $q_{t}$ in this form satisfies (3.42), cf. the proof of Lemma 5.8 in [7]. Then, cf. (2.15),

$$
\left\langle\left\langle G, q_{t}\right\rangle\right\rangle=\int_{\Gamma_{0}}(K G)(\eta)\left(S^{\dagger}(t) R_{0}^{\Lambda, N}\right)(\eta) \lambda(d \eta) \geq 0,
$$

which yields (3.43). The inequality in (3.46) follows by the fact that the semigroup $S^{\dagger}$ is substochastic, see (3.37). This completes the proof.

By (3.41) it follows that $q_{0}^{\Lambda, N} \in \mathcal{K}_{\vartheta_{0}}$, hence we may use it in (3.25) and obtain

$$
k_{t}^{\Lambda, N}=Q_{\vartheta_{1} \vartheta_{0}}^{\sigma}(t) q_{0}^{\Lambda, N}, \quad t \in\left[0, T\left(\vartheta_{1}, \vartheta_{0}\right)\right) .
$$

Proposition 3.10 Let $k_{t}^{\Lambda, N}$ and $q_{t}$ be as in (3.47) and in (3.44), (3.45), respectively. Then, for all $t \in\left[0, T\left(\vartheta_{1}, \vartheta_{0}\right)\right)$, it follows that $k_{t}^{\Lambda, N}=q_{t}$.

Proof A priori $k_{t}^{\Lambda, N}$ and $q_{t}$ lie in different spaces: $\mathcal{K}_{\vartheta_{1}}$ and $\mathcal{G}_{\vartheta}$, respectively. Note that the latter $\vartheta$ can be arbitrary positive. The idea is to construct one more evolution $q_{0}^{\Lambda, N} \mapsto u_{t}$ in some intersection of these two spaces, related to the evolutions in (3.47) and (3.44). Then the proof will follow by the uniqueness as in Proposition 3.9.

For $\vartheta \in \mathbb{R}, \varphi_{\sigma}$ as in (3.23) and $u: \Gamma_{0} \rightarrow \mathbb{R}$, we set, cf. (2.13) and (2.6),

$$
\|u\|_{\sigma, \vartheta}=\operatorname{ess}_{\sup _{\eta \in \Gamma_{0}}} \frac{|u(\eta)| \exp (-\vartheta|\eta|)}{e\left(\eta ; \varphi_{\sigma}\right)}, \quad e\left(\eta ; \varphi_{\sigma}\right):=\prod_{x \in \eta} \varphi_{\sigma}(x)
$$


and then $\mathcal{U}_{\sigma, \vartheta}:=\left\{u: \Gamma_{0} \rightarrow \mathbb{R}:\|u\|_{\sigma, \vartheta}<\infty\right\}$. Clearly,

$$
\mathcal{U}_{\sigma, \vartheta} \hookrightarrow \mathcal{K}_{\vartheta}, \quad \vartheta \in \mathbb{R},
$$

since $\|u\|_{\vartheta} \leq\|u\|_{\sigma, \vartheta}$. Moreover, as in (2.14) we have that $\mathcal{U}_{\sigma, \vartheta} \hookrightarrow \mathcal{U}_{\sigma, \vartheta^{\prime}}$ for $\vartheta^{\prime}>\vartheta$. Let $L^{\Delta, \sigma}$ be defined as in (2.9) with $b$ replaced by $b_{\sigma}$. Then we define an unbounded linear operator $L_{\vartheta, u}^{\Delta, \sigma}: \mathcal{D}_{\vartheta, u}^{\Delta, \sigma} \rightarrow \mathcal{U}_{\sigma, \vartheta}$ with the action as just described and the domain

$$
\mathcal{D}_{\vartheta, u}^{\Delta, \sigma}=\left\{u \in \mathcal{U}_{\sigma, \vartheta}: L^{\Delta, \sigma} u \in \mathcal{U}_{\sigma, \vartheta}\right\}
$$

Clearly, $\mathcal{U}_{\sigma, \vartheta} \subset \mathcal{D}_{\vartheta, u}^{\Delta, \sigma}$ for each $\vartheta^{\prime \prime}<\vartheta$. By (3.33) and (3.40) it follows that $q_{0}^{\Lambda, N}(\eta)=0$ if $|\eta|>N$ or if $\eta$ is not contained in $\Lambda$. Then $q_{0}^{\Lambda, N}$ lies in each $\mathcal{U}_{\sigma, \vartheta^{\prime \prime}}$, $\vartheta^{\prime \prime} \in \mathbb{R}$, and hence in the domain of $L_{\vartheta, u}^{\Delta, \sigma}$ given in (3.50). Thus, we can consider

$$
\frac{d}{d t} u_{t}=L_{\vartheta, u}^{\Delta, \sigma} u_{t},\left.\quad u_{t}\right|_{t=0}=q_{0}^{\Lambda, N}
$$

As in (3.1) we write $L_{\vartheta, u}^{\Delta, \sigma}=A^{\sigma, u}+B^{\sigma, u}$, where $A^{\sigma, u}$ is the multiplication operator by $-E(\eta)$. The operator norm of $B^{\sigma, u}$ can be estimated as follows. By (3.48) we have

$$
|u(\eta)| \leq\|u\|_{\sigma, \vartheta} \exp (\vartheta|\eta|) \prod_{x \in \eta} \varphi_{\sigma}(x)
$$

which yields

$$
\left|\left(B^{\sigma, u} u\right)(\eta)\right| \leq\|u\|_{\sigma, \vartheta}|\eta| \exp (\vartheta|\eta|)\left(\|b\| e^{-\vartheta}+\langle a\rangle e^{\vartheta}\right) \prod_{x \in \eta} \varphi_{\sigma}(x) .
$$

Hence, the operator norm of $B_{\vartheta^{\prime} \vartheta}^{\sigma, u} \in \mathcal{L}\left(\mathcal{U}_{\sigma, \vartheta}, \mathcal{U}_{\sigma, \vartheta^{\prime}}\right)$ satisfies

$$
\left\|B_{\vartheta^{\prime}, \vartheta}^{\sigma, u}\right\| \leq \frac{\|b\| e^{-\vartheta}+\langle a\rangle e^{\vartheta^{\prime}}}{e\left(\vartheta^{\prime}-\vartheta\right)}
$$

which coincides with that in (3.2). Then we repeat the construction made in Propositions 3.1, 3.2 and Lemma 3.3 and obtain the solution of (3.51) in the form

$$
u_{t}=Q_{\vartheta_{1} \vartheta_{0}}^{\sigma, u}(t) q_{0}^{\Lambda, N}, \quad t \in\left[0, T\left(\vartheta_{1}, \vartheta_{0}\right)\right)
$$

where $T\left(\vartheta_{1}, \vartheta_{0}\right)$ is as in (3.9) whereas $Q_{\vartheta_{1} \vartheta_{0}}^{\sigma, u}(t)$ satisfies, cf. (3.11) and Remark 3.4,

$$
\frac{d}{d t} Q_{\vartheta_{1} \vartheta_{0}}^{\sigma, u}(t)=\left(A_{\vartheta_{1} \vartheta^{\prime}}^{\sigma, u}+B_{\vartheta_{1} \vartheta^{\prime}}^{\sigma, u}\right) Q_{\vartheta^{\prime} \vartheta_{0}}^{\sigma, u}(t)=L_{\vartheta_{1}, u}^{\Delta, \sigma} Q_{\vartheta_{1} \vartheta_{0}}^{\sigma, u}(t)
$$


Since $\left(L_{\vartheta_{1}, u}^{\Delta, \sigma}, \mathcal{D}_{\vartheta_{1}, u}^{\Delta, \sigma}\right) \subset\left(L_{\vartheta_{1}}^{\Delta, \sigma}, \mathcal{D}_{\vartheta_{1}}^{\Delta, \sigma}\right)$, and in view of (3.24) and (3.47), (3.49), we have that

$$
\forall t \in\left[0, T\left(\vartheta_{1}, \vartheta_{0}\right)\right) \quad k_{t}^{\sigma}=u_{t} .
$$

On the other hand, for $\vartheta>0$ and $u \in \mathcal{U}_{\sigma, \vartheta^{\prime}}$, by (3.48) we get

$$
\begin{aligned}
& \int_{\Gamma_{0}}|u(\eta)| e^{\vartheta|\eta|} \lambda(d \eta) \leq\|u\|_{\sigma, \vartheta^{\prime}} \int_{\Gamma_{0}} \exp \left(\left(\vartheta^{\prime}+\vartheta\right)|\eta|\right) e\left(\eta ; \varphi_{\sigma}\right) \lambda(d \eta) \\
& =\|u\|_{\sigma, \vartheta^{\prime}} \exp \left(\left\langle\varphi_{\sigma}\right\rangle e^{\vartheta+\vartheta^{\prime}}\right), \quad\left\langle\varphi_{\sigma}\right\rangle:=\int_{\mathbb{R}^{d}} \varphi_{\sigma}(x) d x .
\end{aligned}
$$

Thus, $\mathcal{U}_{\sigma, \vartheta} \hookrightarrow \mathcal{G}_{\vartheta}$ for each $\vartheta^{\prime} \in \mathbb{R}$ and $\vartheta \geq 0$. Likewise, one shows that $\mathcal{D}_{\vartheta^{\prime}, u}^{\Delta, \sigma} \hookrightarrow \widehat{\mathcal{D}} \vartheta$, see (3.39). Since the action of $\widehat{L}$ coincides with that of $L^{\Delta, \sigma}$, by the latter embedding we have that $\left(L_{\vartheta_{1}, u}^{\Delta, \sigma}, \mathcal{D}_{\vartheta_{1}, u}^{\Delta, \sigma}\right) \subset\left(\widehat{L}_{\vartheta}, \widehat{\mathcal{D}}_{\vartheta}\right)$, holding for each $\vartheta>0$. Then by the uniqueness stated in Proposition 3.9 we conclude that $q_{t}=u_{t}$ for all $t \in\left[0, T\left(\vartheta_{1}, \vartheta_{0}\right)\right)$. In view of (3.52), this yields $k_{t}^{\Lambda, N}=u_{t}$, which completes the proof.

\subsubsection{Proof of Lemma 3.5}

We have to show that the assumption of Proposition 3.6 holds true for each $\sigma>0$, which is equivalent to proving that $k_{t}^{\sigma}$ given in (3.25) has the property

$$
\left\langle\left\langle G_{0}, k_{t}^{\sigma}\right\rangle\right\rangle \geq 0
$$

holding for all $t<T\left(\vartheta_{1}, \vartheta_{0}\right)$ and $G_{0} \in B_{\mathrm{bs}}^{\star}\left(\Gamma_{0}\right)$. By definition, a cofinal sequence of $\left\{\Lambda_{n}\right\}_{n \in \mathbb{N}}$ is a sequence of compact subsets $\Lambda_{n} \subset \mathbb{R}^{d}$ such that $\Lambda_{n} \subset \Lambda_{n+1}, n \in \mathbb{N}$, and each $x \in \mathbb{R}^{d}$ is contained in a certain $\Lambda_{n}$. Let $\left\{\Lambda_{n}\right\}_{n \in \mathbb{N}}$ be such a sequence. Fix $\sigma>0$ and then, for given $\Lambda_{n}$ and $N \in \mathbb{N}$, obtain $q_{0}^{\Lambda_{n}, N}$ from $k_{\mu_{0}} \in \mathcal{K}_{\vartheta_{0}}$ by (3.33), (3.40). As in [1, Appendix] one can show that, for each $G \in \mathcal{G}_{\vartheta_{0}}$, the following holds

$$
\lim _{n \rightarrow+\infty} \lim _{N \rightarrow+\infty}\left\langle\left\langle G, q_{0}^{\Lambda_{n}, N}\right\rangle\right\rangle=\left\langle\left\langle G, k_{\mu_{0}}\right\rangle\right\rangle .
$$

Let $G_{0}$ be as in (3.53) and hence lie in any $\mathcal{G}_{\vartheta}$. For $t \in\left[0, T\left(\vartheta_{1}, \vartheta_{0}\right)\right)$ and $k_{t}^{\Lambda_{n}, N}$ as in (3.47), by (3.26) we get

$$
\left\langle\left\langle G_{0}, k_{t}^{\Lambda_{n}, N}\right\rangle\right\rangle=\left\langle\left\langle H_{\vartheta_{0} \vartheta_{1}}^{\sigma}(t) G_{0}, q_{0}^{\Lambda_{n}, N}\right\rangle\right\rangle=:\left\langle\left\langle G, q_{0}^{\Lambda_{n}, N}\right\rangle\right\rangle \geq 0 .
$$

The latter inequality follows by Proposition 3.10 and (3.46). Then (3.53) follows by (3.54) and (3.55).

\subsection{Proof of Theorem 2.4}

To complete proving the theorem we have to construct the continuation of the solution (3.15) to all $t \geq 0$ and prove the upper bound in (2.27). The lower bound follows 
by the fact that $k_{t} \in \mathcal{K}^{\star}$. This will be done by comparing $k_{t}$ with the solution of the equation (2.8) for the Surgailis model given in (2.21). If we denote the latter by $v_{t}$, then

$$
v_{t}(\eta)=\left(W(t) k_{\mu_{0}}\right)(\eta):=\sum_{\xi \subset \eta} e\left(\xi ; \phi_{t}\right) e\left(\eta \backslash \xi ; \psi_{t}\right) k_{\mu_{0}}(\eta \backslash \xi)
$$

For $k_{\mu_{0}} \in \mathcal{K}_{\vartheta_{0}}$, by (2.13) and (1.7) we get from the latter

$$
v_{t}(\eta) \leq\left\|k_{\mu_{0}}\right\|_{\vartheta_{0}} \exp \left\{\vartheta_{0}+\log \left(1+t\|b\| e^{-\vartheta_{0}}\right)\right\}
$$

which holds also in the case $m \equiv 0$. Thus, for a given $T>0, W(t)$ with $t \in[0, T]$ acts as a bounded operator $W_{\vartheta_{T} \vartheta_{0}}(t)$ from $\mathcal{K}_{\vartheta_{0}}$ to $\mathcal{K}_{\vartheta_{T}}$ with

$$
\vartheta_{T}:=\vartheta_{0}+\log \left(1+T\|b\| e^{-\vartheta_{0}}\right) .
$$

For $\vartheta \in \mathbb{R}$, we set, cf. (3.9),

$$
\tau(\vartheta)=T(\vartheta+1, \vartheta)=\left[\|b\| e^{-\vartheta}+\langle a\rangle e^{\vartheta}\right]^{-1} .
$$

For $\vartheta_{1}=\vartheta_{0}+1$, let $k_{t}$ be given in (3.15) with $t \in\left[0, \tau\left(\vartheta_{0}\right)\right)$. Fix some $\kappa \in(0,1 / 2)$ and set $T_{1}=\kappa \tau\left(\vartheta_{0}\right)$. By Lemmas 3.3 and 3.5 we know that $k_{t}=Q_{\vartheta_{1} \vartheta_{0}}(t) k_{\mu_{0}}$ exists and lies in $\mathcal{K}_{\vartheta_{1}}^{\star}$ for all $t \in\left[0, T_{1}\right]$. Take $\vartheta \in\left(\vartheta_{0}, \vartheta_{0}+1\right)$ such that $T_{1}<T\left(\vartheta, \vartheta_{0}\right)$. Then take $\vartheta^{\prime}>\vartheta$ and set, cf. (3.58),

$$
\tilde{\vartheta}_{1}=\max \left\{\vartheta_{0}+1 ; \vartheta^{\prime}+\log \left(1+T_{1}\|b\| e^{-\vartheta^{\prime}}\right)\right\} .
$$

For $t \in\left[0, T_{1}\right]$, we have

$$
v_{t}-k_{t}=\int_{0}^{t} W_{\tilde{\vartheta}_{1} \vartheta^{\prime}}(t-s) D_{\vartheta^{\prime} \vartheta} k_{s} d s
$$

where $k_{s}$ belongs to $\mathcal{K}_{\vartheta}$, whereas $v_{t}$ and $k_{t}$ belong to $\mathcal{K}_{\tilde{\vartheta}_{1}}$. By (3.56) and (3.1) the action of $D$ in (3.60) is

$$
(D k)(\eta)=\left(\sum_{x \in \eta} \sum_{y \in \eta \backslash x} a(x-y)\right) k(\eta)+\int_{\mathbb{R}^{d}}\left(\sum_{y \in \eta \backslash x} a(x-y)\right) k(\eta \cup x) d x,
$$

hence, $v_{t}(\eta)-k_{t}(\eta) \geq 0$ for $\lambda$-almost all $\eta \in \Gamma_{0}$ since $W(t)$ is positive, see (3.56) and (2.22), and $k_{s} \in \mathcal{K}_{\vartheta}^{\star} \subset \mathcal{K}_{\vartheta}^{+}$, see (2.19), (2.20), and Lemma 3.5. Since $k_{t}$ in (3.60) is in $\mathcal{K}^{\star}$, we have that

$$
0 \leq k_{t} \leq v_{t}, \quad t \in\left[0, T_{1}\right],
$$


which by (3.57) yields $k_{t} \in \mathcal{K}_{\vartheta_{T_{1}}}$ and the bound in (2.27) for such $t$, see (3.58). Set $T_{2}=\kappa \tau\left(\vartheta_{T_{1}}\right), \vartheta_{2}=\vartheta_{T_{1}}+1$ and consider $k_{t}^{(2)}=Q_{\vartheta_{2} \vartheta_{T_{1}}}(t) k_{T_{1}}$ with $t \in\left[0, T_{2}\right]$. Clearly, $k_{t}^{(2)}=k_{T_{1}+t}$ for $T_{1}+t<T\left(\vartheta_{0}+1, \vartheta\right)$, see (3.13), and hence is a continuation of $k_{t}$ to $\left[T_{1}, T_{2}\right]$. Now we repeat this procedure due times and obtain

$$
k_{t}^{(n)}=Q_{\vartheta_{n} \vartheta_{T_{n-1}}}(t) k_{T_{n-1}},
$$

where $\vartheta_{n}=\vartheta_{T_{n-1}}+1$ and

$$
\begin{aligned}
T_{n} & =\kappa \tau\left(\vartheta_{T_{n-1}}\right) \\
\vartheta_{T_{n}} & =\vartheta_{T_{n-1}}+\log \left(1+T_{n-1}\|b\| e^{-\vartheta_{T_{n}-1}}\right), \quad \vartheta_{T_{0}}:=\vartheta_{0} .
\end{aligned}
$$

The continuation to all $t>0$ will be obtained if we show that $\sum_{n \geq 1} T_{n}=+\infty$. Assume that this is not the case. From the second line in (3.62) we get $T_{n-1}=$ $\left(e^{\vartheta T_{n}}-e^{\vartheta T_{n-1}}\right) /\|b\|$. Hence

$$
\sum_{n=1}^{N} T_{n}=\left(e^{\vartheta} T_{N}-e^{\vartheta_{0}}\right) /\|b\| .
$$

Thus, the mentioned series converges if the sequence $\left\{\vartheta_{T_{n}}\right\}_{n \in \mathbb{N}}$ is bounded, say by $\bar{\vartheta}$. However, in this case one cannot get $T_{n} \rightarrow 0$ as $n \rightarrow+\infty$, for it contradicts the first line in (3.62) since

$$
\tau(\vartheta) \geq\left[e\langle a\rangle e^{\bar{\vartheta}}+\|b\| e^{-\vartheta_{0}}\right]^{-1}
$$

see (3.59). Clearly, the upper bound in (3.61) holds on each $\left[T_{n-1}, T_{n}\right]$. This completes the proof.

\section{The global boundedness}

Here we prove Theorem 2.5. In the nontrivial case $a(0)>0$, see Assumption 1.1, let $\Delta$ be a cubic cell containing the origin such that

$$
\inf _{x \in \Delta} a(x)=: a_{\Delta}>0,
$$

which is possible since $a$ is continuous. For $\eta$ contained in a translate of $\Delta,|\eta| \geq 2$, and $x \in \eta$, we then have

$$
\sum_{y \in \eta \backslash x} a(x-y) \geq a_{\Delta}(|\eta|-1) \geq a_{\Delta} .
$$

For a translate of $\Delta$, we consider the observables $N_{\Delta}^{n}: \Gamma \rightarrow \mathbb{N}_{0}$ defined as follows: $N_{\Delta}^{n}(\gamma)=\left|\gamma_{\Delta}\right|^{n}, n \in \mathbb{N}$. Then 


$$
\begin{aligned}
& N_{\Delta}(\gamma)=\sum_{x \in \gamma} I_{\Delta}(x), \\
& N_{\Delta}^{n}(\gamma)=\sum_{l=1}^{n} l ! S(n, l) \sum_{\left\{x_{1}, \ldots, x_{l}\right\} \subset \gamma} I_{\Delta}\left(x_{1}\right) \cdots I_{\Delta}\left(x_{l}\right), \quad n \geq 2,
\end{aligned}
$$

where $I_{\Delta}$ is the indicator function of $\Delta$ and $S(n, l)$ is a Stirling numbers of the second kind, equal to the number of distinct ways of dividing $n$ labeled items into $l$ unlabeled groups. It has the following representation, cf. [13],

$$
S(n, l)=\frac{1}{l !} \sum_{s=0}^{l}(-1)^{l-s}\left(\begin{array}{l}
l \\
s
\end{array}\right) s^{n}
$$

Then, for $\mu \in \mathcal{P}_{\exp }(\Gamma)$, by (2.6) we have that

$$
\mu\left(N_{\Delta}^{n}\right)=\sum_{l=1}^{n} S(n, l) \int_{\Delta} \cdots \int_{\Delta} k_{\mu}^{(l)}\left(x_{1}, \ldots, x_{l}\right) d x_{1} \cdots d x_{l} .
$$

For $l \in \mathbb{N}$, we set

$$
\begin{aligned}
F_{\Delta}^{(l)}(\gamma) & =\sum_{\left\{x_{1}, \ldots, x_{l}\right\} \subset \gamma} I_{\Delta}\left(x_{1}\right) \cdots I_{\Delta}\left(x_{l}\right) \\
& =\frac{1}{l !} N_{\Delta}(\gamma)\left(N_{\Delta}(\gamma)-1\right) \cdots\left(N_{\Delta}(\gamma)-l+1\right)
\end{aligned}
$$

And also $F_{\Delta}^{(0)}(\gamma) \equiv 1$. Then we can rewrite (4.3) as follows

$$
N_{\Delta}^{n}(\gamma)=\sum_{l=1}^{n} l ! S(n, l) F_{\Delta}^{(l)}(\gamma)
$$

An easy calculation yields

$$
F_{\Delta}^{(l)}(\gamma \cup x)-F_{\Delta}^{(l)}(\gamma)=I_{\Delta}(x) F_{\Delta}^{(l-1)}(\gamma),
$$

For $\mu_{t}$ as in Theorem 2.4, we set

$$
q_{\Delta}^{(0)}(t) \equiv 1, \quad q_{\Delta}^{(l)}(t)=\mu_{t}\left(F_{\Delta}^{(l)}\right), \quad l \in \mathbb{N} .
$$

By (4.5), (4.6) and (4.7) it follows that

$$
q_{\Delta}^{(l)}(0)=\frac{1}{l !} \int_{\Delta} \ldots \int_{\Delta} k_{\mu_{0}}^{(l)}\left(x_{1}, \ldots, x_{l}\right) d x_{1} \cdots d x_{l} .
$$

Since $\mu_{0}$ is in $\mathcal{P}_{\exp }(\Gamma)$, one finds $\vartheta \in \mathbb{R}$ such that $k_{\mu_{0}}^{(l)}\left(x_{1}, \ldots, x_{l}\right) \leq e^{\vartheta}$, cf. (2.3). By (4.10) this yields 


$$
q_{\Delta}^{(l)}(0) \leq\left[\mathrm{V}(\Delta) e^{\vartheta}\right]^{l} / l !, \quad l \in \mathbb{N} .
$$

Recall that $a_{\Delta}$ is defined in (4.1), see also (4.2). Set

$$
b_{\Delta}=\int_{\Delta} b(x) d x, \quad \kappa_{\Delta}=\max \left\{\mathrm{V}(\Delta) e^{\vartheta} ; b_{\Delta} / a_{\Delta}\right\}
$$

where $\vartheta$ is as in (4.11).

The proof of the lemma below is based on the following elementary arguments. Let $u:[0,+\infty) \rightarrow \mathbb{R}$ be continuously differentiable with the derivative satisfying

$$
u^{\prime}(t) \leq v_{0}-v_{1} u(t), \quad v_{0}, v_{1}>0
$$

Then by standard arguments one obtains that

$$
u(t) \leq u(0) e^{-v_{1} t}+\frac{v_{0}}{v_{1}}\left(1-e^{-v_{1} t}\right), \quad t \geq 0,
$$

which, in particular, means that

$$
u(t) \leq \max \left\{u(0) ; v_{0} / v_{1}\right\}
$$

and also: for each $\varepsilon>0$, there exists $\tau_{\varepsilon} \geq 0$ such that

$$
\forall t \geq \tau_{\varepsilon} \quad u(t) \leq \varepsilon+v_{0} / v_{1}
$$

Lemma 4.1 Let $\Delta$ be as in (4.1) and $\mu_{t}, t \geq 0$ be as in Theorem 2.4, and hence $q_{\Delta}^{(l)}(0)$ satisfies (4.11) with some $\vartheta$. Let $\kappa_{\Delta}$ be as in (4.12) for these parameters. Then

$$
\forall t \geq 0 \quad q_{\Delta}^{(l)}(t) \leq \kappa_{\Delta}^{l} / l !, \quad l \in \mathbb{N}
$$

Proof By (1.4) we have that

$$
\frac{d}{d t} q_{\Delta}^{(l)}(t)=\mu_{t}\left(L F_{\Delta}^{(l)}\right)
$$

which by means of (4.8) can be written

$$
\begin{aligned}
\frac{d}{d t} q_{\Delta}^{(l)}(t) & =b_{\Delta} q_{\Delta}^{(l-1)}(t)-\int_{\Gamma}\left(\sum_{x \in \gamma_{\Delta}}\left(\sum_{y \in \gamma \backslash x} a(x-y)\right) F_{\Delta}^{(l-1)}(\gamma \backslash x)\right) \mu_{t}(d \gamma) \\
& \leq b_{\Delta} q_{\Delta}^{(l-1)}(t)-\int_{\Gamma}\left(\sum_{x \in \gamma_{\Delta}}\left(\sum_{y \in \gamma_{\Delta} \backslash x} a(x-y)\right) F_{\Delta}^{(l-1)}(\gamma \backslash x)\right) \mu_{t}(d \gamma) \\
& \leq b_{\Delta} q_{\Delta}^{(l-1)}(t)-a_{\Delta} \int_{\Gamma}\left(\sum_{x \in \gamma_{\Delta}} F_{\Delta}^{(l-1)}(\gamma \backslash x)\right) \mu_{t}(d \gamma)
\end{aligned}
$$


By (4.7) it follows that

$$
\sum_{x \in \gamma_{\Delta}} F_{\Delta}^{(l-1)}(\gamma \backslash x)=l F_{\Delta}^{(l)}(\gamma) .
$$

We apply this in (4.17) and obtain, cf. (4.13) and (4.12),

$$
\frac{d}{d t} q_{\Delta}^{(l)}(t) \leq b_{\Delta} q_{\Delta}^{(l-1)}(t)-l a_{\Delta} q_{\Delta}^{(l)}(t), \quad l \in \mathbb{N} .
$$

For $l=1$, by (4.9) and (4.14) we get from the latter that (4.16) holds. Now we assume that (4.16) holds for a given $l-1$. It yields in (4.18)

$$
\frac{d}{d t} q_{\Delta}^{(l)}(t) \leq \frac{b_{\Delta} \kappa_{\Delta}^{l-1}}{(l-1) !}-l a_{\Delta} q_{\Delta}^{(l)}(t),
$$

from which by (4.14) we obtain that (4.16) holds also for $l$.

Proof of Theorem 2.5 By means of the evident monotonicity

$$
\mu\left(N_{\Lambda}^{n}\right) \leq \mu\left(N_{\Lambda}^{n+1}\right), \quad \mu\left(N_{\Lambda}^{n}\right) \leq \mu\left(N_{\Lambda^{\prime}}^{n}\right), \quad \text { for } \Lambda \subset \Lambda^{\prime},
$$

we conclude that it is enough to prove the statement for: (a) $n=2^{s}$; (b) $\Lambda$ being a finite sum of the disjoint translates of the cubic cell $\Delta$ as in Lemma 4.1. Let $m$ be such that

$$
\Lambda=\bigcup_{l=1}^{m} \Delta_{l}
$$

By the estimate

$$
\left(\sum_{l=1}^{n} a_{l}\right)^{2} \leq n \sum_{l=1}^{n} a_{l}^{2}
$$

we prove that

$$
N_{\Lambda}^{2^{s}}(\gamma) \leq m^{2^{s}-1} \sum_{l=1}^{m} N_{\Delta_{l}}^{2^{s}}(\gamma), \quad s \in \mathbb{N}_{0} .
$$

Then by Lemma 4.1 and (4.7) we obtain

$$
\mu_{t}\left(N_{\Lambda}^{2^{s}}\right) \leq m^{2^{s}} T_{2^{s}}\left(\bar{\kappa}_{\Delta}\right)=[\mathrm{V}(\Lambda)]^{2^{s}}\left(T_{2^{s}}\left(\bar{\kappa}_{\Delta}\right) /[\mathrm{V}(\Delta)]^{2^{s}}\right)
$$

where $T_{n}$ is the Touchard polynomial

$$
T_{n}(\varkappa):=\sum_{l=1}^{n} S(n, l) \varkappa^{l}
$$


with $S(n, l)$ given in (4.4), see [13, Eq. (3.4)], and

$$
\bar{\kappa}_{\Delta}:=\mathrm{V}(\Delta) \max \left\{e^{\vartheta} ;\|b\| / a_{\Delta}\right\},
$$

cf. (4.12). This proves (2.28).

Like in [4], it is possible to show that if the initial state $\mu_{0}$ is such that each $k_{\mu_{0}}^{(l)} \in C_{\mathrm{b}}\left(\left(\mathbb{R}^{d}\right)^{l}\right)$ - the set of bounded continuous functions, then so is $k_{t}^{(l)}$ for all $t>0$. As in (2.25) we have, see also (4.5),

$$
\mu_{t}\left(N_{\Delta}\right)=\int_{\Delta} k_{t}^{(1)}(x) d x .
$$

By taking a sequence of $\Delta$ shrinking up to a given $x$ and applying (4.16) we obtain

$$
k_{t}^{(1)}(x) \leq \max \left\{k_{\mu_{0}}^{(1)}(x) ; b(x) / a(0)\right\} \leq \max \left\{\left\|k_{\mu_{0}}^{(0)}\right\|_{L^{\infty}\left(\mathbb{R}^{d}\right)} ;\|b\| / a(0)\right\},
$$

which proves the bound for $k_{t}^{(1)}$. Let us now prove the validity of the second estimate in (1.8). The bound for $k_{t}^{(2)}(x, x)$ can be obtained from (4.16) in the way similar to that used in getting (4.19). To bound for $k_{t}^{(2)}(x, y)$ with $x \neq y$, let us take two disjoint cells $\Delta_{x}$ and $\Delta_{y}$. Both are of side $h>0$ and such that: (a) $x \in \Delta_{x}$ and $y \in \Delta_{y}$; (b) $\Delta_{x} \rightarrow\{x\}$ and $\Delta_{y} \rightarrow\{y\}$ as $h \rightarrow 0$. Then set

$$
F_{h}(\gamma)=\left[\sum_{z \in \gamma}\left(I_{\Delta_{x}}(z)-I_{\Delta_{y}}(z)\right)\right]^{2}
$$

For the state $\mu_{t}$, we have

$$
0 \leq \mu_{t}\left(F_{h}\right)=q_{\Delta_{x}}^{(2)}(t)+q_{\Delta_{y}}^{(2)}(t)-2 \int_{\Delta_{x}} \int_{\Delta_{y}} k_{t}^{(2)}\left(z_{1}, z_{2}\right) d z_{1} d z_{2} .
$$

By (4.12) and (4.16) this yields

$$
\int_{\Delta_{x}} \int_{\Delta_{y}} k_{t}^{(2)}\left(z_{1}, z_{2}\right) d z_{1} d z_{2} \leq \frac{1}{2} \max \left\{\kappa_{\Delta_{x}}^{2} ; \kappa_{\Delta_{y}}^{2}\right\} \leq \frac{h^{2 d}}{2} \max \left\{e^{2 \vartheta} ;\left(\|b\| / a_{h}\right)^{2}\right\},
$$

where $a_{h}:=\min \left\{a_{\Delta_{x}} ; a_{\Delta_{y}}\right\}$. Passing here to the limit $h \rightarrow 0$ and taking into account the assumed continuity of $k_{t}^{(2)}$ and $a$ we obtain the second inequality in (1.8). This completes the proof.

Note that the smaller bound in (4.19) coincides with the corresponding bound in the exactly soluble Surgailis model in which the mortality rate $m(x)$ is substituted by $a(0)$. That is, the competition here amounts to the appearance of an effective mortality $a(0)$. Another important observation regarding the competition in this model is based on (4.15). Let $\Lambda$ be compact and $k_{t}^{(1)}$ satisfy (4.19). Then for an arbitrary $\varepsilon>0$, one 
finds $\tau(\varepsilon, \Lambda)$, dependent also on $\mu_{0}$, such that, for all $x \in \Lambda$ and $t \geq \tau(\varepsilon, \Lambda)$, the following holds

$$
k_{t}^{(1)}(x) \leq \frac{b(x)}{a(0)}+\varepsilon .
$$

That is, after some time the density at each point of $\Lambda$ approaches a certain level, independent of the initial distribution of the entities in $\Lambda$.

Acknowledgements The present research was supported by the European Commission under the project STREVCOMS PIRSES-2013-612669 and by the SFB 701 "Spektrale Strukturen and Topologische Methoden in der Mathematik". The authors are also grateful to the referee whose suggestions helped to improve the presentation of the paper.

Open Access This article is distributed under the terms of the Creative Commons Attribution 4.0 International License (http://creativecommons.org/licenses/by/4.0/), which permits unrestricted use, distribution, and reproduction in any medium, provided you give appropriate credit to the original author(s) and the source, provide a link to the Creative Commons license, and indicate if changes were made.

\section{References}

1. Berns, Ch., Kondratiev, Y., Kozitsky, Y., Kutoviy, O.: Kawasaki dynamics in continuum: micro- and mesoscopic descriptions. J. Dyn. Differ. Equ. 25, 1027-1056 (2013)

2. Cox, J.T.: Coalescing random walks and voter model consensus times on the torus in $\mathbb{Z}^{d}$. Ann. Probab. 17, 1333-1366 (1989)

3. Finkelshtein, D., Kondratiev, Y.: Regulation mechanisms in spatial stochastic development models. J. Stat. Phys. 136, 103-115 (2009)

4. Finkelshtein, D.L., Kondratiev, Y., Kozitsky, Y.: Glauber dynamics in continuum: a constructive approach to evolution of states. Discrete Contin. Dyn. Syst. 33, 1431-1450 (2013)

5. Finkelshtein, D.L., Kondratiev, Y., Kozitsky, Y., Kutovyi, O.: The statistical dynamics of a spatial logistic model and the related kinetic equation. Math. Models Methods Appl. Sci. 25, 343-370 (2015)

6. Kingman, J.F.C.: Poisson Processes. Oxford Studies in Probability, vol. 3. The Clarendon Press, New York (1993)

7. Kondratiev, Y, Kozitsky, Y.: The evolution of states in a spatial population model. J. Dyn. Differ. Equ. (2016). doi:10.1007/s10884-016-9526-6

8. Kondratiev, Y., Kuna, T.: Harmonic analysis on configuration space. I. General theory. Infin. Dimens. Anal. Quantum Probab. Relat. Top. 5, 201-233 (2002)

9. Kondratiev, Y., Kuna, T., Oliveira, M.J.: Holomorphic Bogoliubov functionals for interacting particle systems in continuum. J. Funct. Anal. 238, 375-404 (2006)

10. Kozitsky, Y.: Dynamics of spatial logistic model: finite systems. In: Banasiak, J., Bobrowski, A., Lachowicz, M. (eds.) Semigroups of Operators-Theory and Applications: Będlewo, Poland, October 2013. Springer Proceedings in Mathematics \& Statistics 113, Springer, pp. 197-211 (2015)

11. Murrell, D.J., Dieckmann, U., Law, R.: On moment closures for population dynamics in contunuous space. J. Theor. Biol. 229, 421-432 (2004)

12. Nazin, G.I.: Method of the generating functional. J. Sov. Math. 31, 2859-2886 (1985)

13. Riordan, J.: Moment recurrence relations for binomial, Poisson and hypergeomentric frequency distributions. Ann. Math. Stat. 8, 103-111 (1937)

14. Surgailis, D.: On multiple Poisson stochastic integrals and associated Markov semigroups. Probab. Math. Stat. 3(2), 217-239 (1984) 